\title{
Cyclische Bis(amino)-arsa-, -stiba-, bismachloride und ein spezielles Tris(amino)bismutan
}

\author{
M. VEITH* und B. BerTsch \\ Saarbrücken, Institut für Anorganische Chemie der Universität des Saarlandes
}

Inhaltsübersicht. In den Trichloriden von Arsen, Antimon und Bismut lassen sich jeweils zwei Chloratome durch den Liganden $-\mathrm{N}\left({ }^{\mathrm{t}} \mathrm{Bu}\right)-\mathrm{SiMe}_{2}-\mathrm{N}\left({ }^{\mathrm{t}} \mathrm{Bu}\right)$ - austauschen, wodurch viergliedrige Ringe $\left(\mathrm{SiN}_{2} \mathrm{El}\right)$ entstehen. Sowohl in Lösung (temperatur- und konzentrationsabhängige ${ }^{\mathbf{1}} \mathrm{H}-\mathrm{NMR}$ Spektren) als auch im Festkörper (Röntgenstrukturanalysen) besitzen die Verbindungen $\mathbf{3}(\mathrm{EI}=\mathrm{As}$ ), $4(\mathrm{El}=\mathrm{Sb})$ bzw. $5(\mathrm{El}=\mathrm{Bi})$ unterschiedliche Assoziationsgrade bzw. Strukturen. Das Arsenderivat 3 kristallisiert monoklin, in der Raumgruppe $\mathrm{P}{ }_{1} / \mathrm{c}$ mit 4 Formeleinheiten pro Elementarzelle $(\mathrm{a}=$ $\left.920,0(6), \mathrm{b}=1462,9(9), \mathrm{e}=1240,8(7) \mathrm{pm}, \beta=105,8(1)^{\circ}\right)$, das Antimon- und Bismutderivat 4 und 5) sind isotyp und isostrukturell und kristallisieren ortho-rhombisch (Raumgruppe Pnma, $Z=4$; Gitterkonstanten für 4: $a=1140,2(8), b=1095,7(7), c=1328,7(9)$ pm; für $5: a=1105,1(5)$,

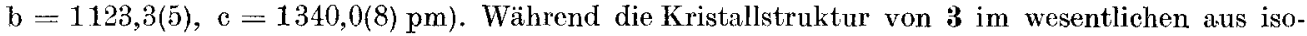
lierten Molekülen besteht, die eine schwache Tendenz zu paarweiser Assoziation besitzen (As-Cl $\cdots$ As Brücken, $\left.\mathrm{As}-\mathrm{Cl}=234, \bar{b}(1), \mathrm{Cl} \cdots \mathrm{As}_{\mathrm{s}}=442,3(1) \mathrm{pm}\right)$, sind die Moleküle in den Kristallen von 4 und 5 eindimensional verkettet. Die zu den $\mathrm{SbN}_{2} \mathrm{Si}$ - bzw. $\mathrm{BiN}_{2} \mathrm{Si}$-Ringen nahezu senkrecht stehenden Chlorsubstituenten treten mit Nachbarmolekülen derart in Wechselwirkung, daß jedes Sb- und BiAtom vierfach koordiniert wird und eine unendiche $\mathrm{El}-\mathrm{Cl} \cdots \mathrm{El}$-Cl-Kette entsteht, die an den Chloratomen gewinkelt ist (4): $143,5^{\circ},(\mathbf{5}): 145,7^{\circ}$. Die verbrückende Rolle des Chloratoms ist bei der Bi-Verbindung stärker ausgeprägt als bei der Sb-Verbindung $(\mathrm{Sb}-\mathrm{Cl}=247,2(3), \mathrm{Cl} \cdots \mathrm{Sb}=$ $352,1(3) \mathrm{pm} ; \mathrm{Bi}-\mathrm{Cl}=274,8(4), \mathrm{Cl} \cdots \mathrm{Bi}=304,7(4) \mathrm{pm})$. Als Nebenprodukt kann bei der Darstellung von 5 , eine Verbindung 6 erhalten werden, die nur Stickstoffatome am Bismut gebunden hat: $\mathrm{Me}_{2} \mathrm{Si}\left(\mathrm{N}^{\mathrm{t}} \mathrm{Bu}\right)_{2} \mathrm{Bi}-\mathrm{N}\left({ }^{\mathrm{t}} \mathrm{Bu}\right)-\mathrm{SiMe}_{2}-\left({ }^{\mathrm{t}} \mathrm{Bu}\right) \mathrm{N}-\mathrm{Bi}\left(\mathrm{N}^{\mathrm{t}} \mathrm{Bu}\right)_{2} \mathrm{SiMe}_{2}$. Diese Verbindung 6 kristallisiert mono$\mathrm{klin}\left(\mathrm{P}_{1}{ }_{1} / \mathrm{e}, \mathrm{a}=1474,2(3), \mathrm{b}=1477,6(3), \mathrm{c}=1997,7(6) \mathrm{pm}, \beta=94,68(8)^{\circ}\right)$. Die Abweichung von der $\mathrm{C}_{2}$-Symmetrie bei 6 (die zweizählige Achse verläuft durch die mittlere $\mathrm{SiMe}_{2}$-Gruppe) ist nur unbedeutend. Die $\mathrm{Bi}-\mathbf{N}$-Bindungslängen innerhalb der $\mathrm{SiN}_{2} \mathrm{Bi}$-Ringe $(\mathrm{Bi}-\mathbf{N}$ (Mittel) $=2 \mathbf{1 6 , 5}(5) \mathrm{pm})$ sind kürzer als die außerhalb $(\mathbf{B} \mathbf{i}-\mathbf{N}($ Mittel $)=220,45(5) \mathrm{pm})$.

\section{Cyclic Bis(amino)-Arsa-, -Stiba-, -Bismachlorides and a Special Tris(amino)bismutane}

Abstract. In the trichlorides of arsenic, antimony, and bismut two of the chlorine atoms have been substituted by the ligand $-\mathrm{N}\left({ }^{\mathrm{t}} \mathrm{Bu}\right)-\mathrm{SiMe}_{2}-\left({ }^{\mathrm{t}} \mathrm{Bu}\right) \mathrm{N}$ - yielding four membered cycles $\left(\mathrm{SiN}_{2} \mathrm{El}\right)$. The kind of association and the structures of the compounds $3(\mathrm{El}=\mathrm{As}), \mathbf{4}(\mathrm{El}=\mathrm{Sb})$, and $5(\mathrm{El}=\mathrm{Bi})$ are different in solution as well as in the solid state as found by ${ }^{1} \mathrm{H}-\mathrm{NMR}$ spectra (temperature and concentration dependancy) and by $\mathrm{X}$-ray structure analyses. The arsenic compound 3 crystallizes in the monoclinic lattice, space group $\mathrm{P}_{1}^{2} / \mathrm{c}$, with 4 molecules within the unit cell $(\mathrm{a}=920.0(6)$, $\left.\mathrm{b}=1.462 .9(9), \mathrm{c}=1240.8(7) \mathrm{pm}, \beta=105.8(1)^{\circ}\right)$, the antimony and bismut derivatives 4 and 5 are isotypic and isostructural and crystallize in the ortho-rhombic system (space group Prma, $Z=4$;

*) jeweils Korrespondenzautor 
lattice constants for $4: a=1140.2(8), b=1095,7(7), c=1328.7(9) \mathrm{pm}$; for 5 ; $a=1105.1(5)$, $\mathrm{b}=1123.3(5), \mathrm{c}=1340.0(8) \mathrm{pm})$. While the crystal structure of 3 contains isolated molecules, which tend weakly to associate to dimers via $\mathrm{As}-\mathrm{Cl} \cdots$ As bridges $(\mathrm{As}-\mathrm{Cl}=234.5(1), \mathrm{Cl} \cdots \mathrm{As}=$ $442.3(1) \mathrm{pm}$ ), the structures of 4 and 5 are filled up with one dimensionally catenated molecules. The chlorine substituents, which are almost perpendicular to the $\mathrm{SbN}_{2} \mathrm{Si}$ and $\mathrm{BiN} \mathrm{N}_{2} \mathrm{Si}$ cycles, interact with neighbouring molecules in such a way that each $\mathrm{Sb}$ and $\mathrm{Bi}$ atom becomes four coordinated. An infinite $\mathrm{El}-\mathrm{Cl} \cdots \mathrm{El}-\mathrm{Cl}$ chain results from these interactions with angles $143.5^{\circ}(4)$ and $145.7^{\circ}(5)$ at the chlorine atoms. The bridging capacity of the chlorine atom is stronger in the Bi-compound compared to the Sb-molecule $(\mathrm{Sb}-\mathrm{Cl}=247.2(3), \mathrm{Cl} \cdots \mathrm{Sb}=352.1(3) \mathrm{pm} ; \mathrm{Bi}-\mathrm{Cl}=274.8(4)$, $\mathrm{Cl} \cdots \mathrm{Bi}=304.7(4) \mathrm{pm})$. As a minor product in the synthesis of 5 a compound 6 may be obtained which has exclusively nitrogen atoms bonded to the bismut:

$\mathrm{Me}_{2} \mathrm{Si}\left(\mathrm{N}^{\mathrm{t}} \mathrm{Bu}\right)_{2} \mathrm{Bi}-\mathbf{N}\left({ }^{\mathrm{t}} \mathrm{Bu}\right)-\mathrm{SiMe}_{2}-\left({ }^{\mathrm{t}} \mathrm{Bu}\right) \mathbf{N}-\mathbf{B i}\left(\mathbf{N}^{\mathrm{t}} \mathrm{Bu}\right)_{2} \mathrm{SiMe}_{2}$.

The compound 6 crystallizes in a monoclinic lattice $(\mathrm{P} 2 / \mathrm{c}, \mathrm{a}=1474.2(3), b=1477.6(3)$, $\left.\mathrm{c}=1997.7(6) \mathrm{pm}, \beta=94.68(8)^{\circ}\right)$. The distortion of the $\mathrm{C}_{2}$-symmetry in 6 (the twofold axis passes within the $\mathrm{Me}_{2} \mathrm{Si}$-group at the center) is of minor importance. The $\mathrm{Bi}-\mathrm{N}$-bond lengths within the $\mathrm{SiN}_{2} \mathrm{Bi}$-cycles $(\mathrm{Bi}-\mathbf{N}$ (averages) $=216.5(5) \mathrm{pm}$ ) are shorter than the corresponding external distances $(\mathrm{Bi}-\mathrm{N}$ (average) $=220.45(5) \mathrm{pm})$.

\section{Einleitung}

Im Zuge unserer Untersuchungen zur Struktur und Chemie von Bis(amino)germylenen, -stannylenen und -plumbylenen (A) [1-3] interessierten wir uns für Verbindungen, die zu A isoelektronisch sind. Die mit Formel B wiedergegebenen Kationen erfüllen formal diese Bedingungen und erschienen uns einer eingehenden Untersuchung wert.

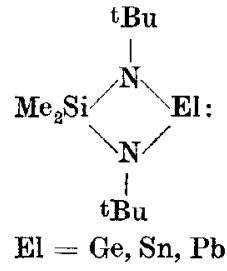

A<smiles>[AlH2][Al]1C=CC=C(Br)N1Cc1ccccc1</smiles>

$\mathrm{El}=\mathrm{As}, \mathrm{Sb}, \mathrm{Bi}$

$\mathbf{B}$

Zur Darstellung des Kations B bietet sich die Möglichkeit an, zunächst das Element der fünften Hauptgruppe in seiner dreiwertigen Stufe in den Vierring einzubauen, um dann in einem zweiten Schritt den überschüssigen Liganden als $\mathrm{X}^{-}$abzuspalten [4]. In der vorliegenden Veröffentlichung beschreiben wir die Synthese der ,Vorstufen", $\mathrm{Me}_{2} \mathrm{Si}\left(\mathrm{N}^{\mathrm{t}} \mathrm{Bu}\right)_{2} \mathrm{El}-\mathrm{X}$, wobei in allen Fällen $\mathrm{X}$ mit Chlor gleichzusetzen ist. Zur Charakterisierung der Produkte benützten wir, außer spektroskopischen Methoden, Röntgenstrukturbestimmungen. Bei der Synthese des Bismutderivates erhielten wir ein weiteres Produkt, das wir ebenfalls chemisch und strukturell charakterisiert haben.

\section{Zur Synthese von cyclischen Bis(amino) -arsa-, -stiba- und -bismutchloriden}

Arsentrichlorid, Antimontrichlorid und Bismuttrichlorid lassen sich problemlos unter Lithiumchloridabspaltung mit dem Dilithiumsalz des Bisaminosilans 1 zu den Zielmolekülen 3, 4 und 5 umsetzen (Gl. (1)), oder können aus 2 Zinn(II)- 
chlorid freisetzen. was ebenfalls gemäß Gl. (ㅇ) zu denselben Produkten führt.

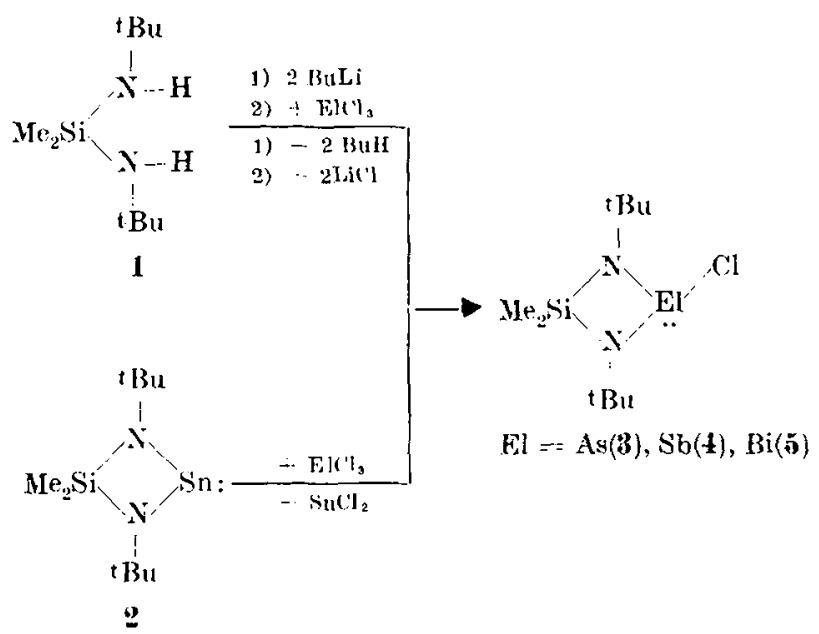

Während die Synthese nach $G$ l. (1) bereits als klassisch anzusehen ist und auch für andere cyclische Derivate in ähnlicher oder abgewandelter Form Verwendung fand [j-..11], bedarf der Syntheseweg (2) eines Kommentares.

Die analoge Cmsetzung von 2 mit Phosphortrichlorid führt nicht zur Zinn(II) chloridbildung, sondern zur Oxidation des Zinn(II) zu Zinn(IV) unter gleichzeitjger Reduktion des Phosphors zur einwertigen Stufe |1.2] (Gl. (3)).

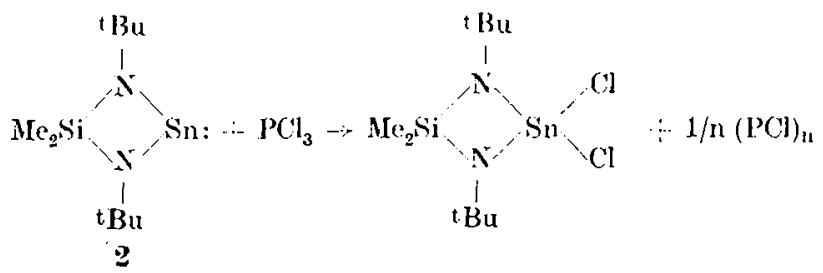

Während diese Reaktion beim Phosphortrichlorid quantitativ verläuft, ist die analoge Umsetzung bei den schwereren Elementtrichloriden (zumindest bei $\mathrm{AsCl}_{3}$ und $\mathrm{SbCl}_{3}$ ) nur noch in Spuren nachzuweisen (laut ${ }^{1} \mathrm{H}-\mathrm{N} M \mathrm{R}$ der Roalitionslösung $5-10 \%$, und bleibt beim $\mathrm{BiCl}_{3}$ völlig aus. Offenbar kommt den im Vergleich zum $\mathrm{P}$ größeren Atomradien von $\mathrm{As}$, $\mathrm{Sb}$ und $\mathrm{Bi}$ und ihren niederen Elektronegativitäten hierbei Bedeutung zu (vgl. auch die Reaktionsreihe $\mathrm{Me}_{2} \mathrm{Si}\left(\mathrm{N}^{\top} \mathrm{Bu}\right)_{2} \mathrm{El}:(\mathrm{El}=$ Ge, Sn und $\mathrm{Pb}$ ) gegenüber $\left.\mathrm{PCl}_{3}[12]\right)$.

Erfolgt die Reinigung von $\mathrm{BiCl}_{3}$ nicht gründlich genug, so befindet sich das Halogenid im molaren Cnterschuß gegenüber dem Dilithiumsalz von 1 . In diesem

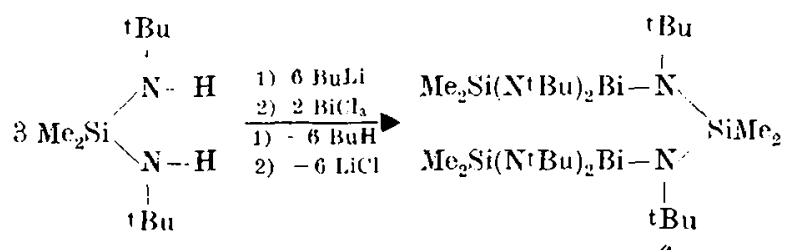


Falle beobachtet man einen anderen Reaktionsverlauf und die Bildung des Produktes 6, in dem die Bismutatome vollständig durch Stickstoffatome substituiert sind (Gl. (4)). 6 kann als Nebenprodukt zusammen mit $\mathbf{5}$ entstehen, was sich durch peinlich sauberes Arbeiten vermeiden läßt, oder entsteht quantitativ bei entsprechend vorgegebenem Molverhältnis.

Alle Produkte 3-6 können durch Umkristallisation in befriedigenden Ausbeuten erhalten werden und liefern richtige Analysenwerte (siehe exp. Teil).

\section{${ }^{1}$ H-NMR-Spektren von 3,4 und 5}

Die beiden tert-Butylgruppen der Verbindungen 3, 4 und 5 haben jeweils gleiche chemische Umgebung - die Moleküle besitzen in Lösung alle $m\left(\mathrm{C}_{\mathrm{s}}\right)$-Symmetrie - und können daher im ${ }^{1} \mathrm{H}$-NMR-Spektrum nicht unterschieden werden. Für die Methylgruppen des Siliciums würde man, auf Grund ihrer unterschiedlichen Stellungen zu den Chlorsubstituenten, zwei Signale erwarten. Bei Raumtemperatur findet man jedoch nur für die Antimonverbindung 4 das erwartete Signalbild, während für die Arsenverbindung 3 ein verbreitertes Signal und für die Bismutverbindung 5 ein scharfes Singulett der Dimethylsilylgruppe zugeordnet werden muß.

Wir haben für alle drei Verbindungen nochmals Spektren in Abhängigkeit von der Konzentration und der Temperatur der Proben aufgenommen. Über Molmassenbestimmungen konnten wir festellen, daß die Moleküle im verwendeten Lösungsmittel (Benzol, Toluol) monomer vorliegen. Sowohl eine Abnahme der Konzentration der Lösung als auch eine Temperaturerniedrigung bei konstanter Konzentration führt zur Aufspaltung des $\mathrm{Me}_{2} \mathrm{Si}$-Signales bei 3 . Ebenso führt eine Konzentrationszunahme oder eire Temperaturerhöhung zur Koaleszenz der $\mathrm{Me}_{2} \mathrm{Si}$-Signale bei der Antimonverbindung 4. Keinerlei Auswirkung haben Konzentrations- bzw. Temperaturänderung auf das Spektrum von 5, das in allen Fällen ein lagekonstantes $\mathrm{Me}_{2} \mathrm{Si}$-Singulett aufweist.

Von den beiden möglichen Prozessen, die innerhalb der NMR-Zeitskala zu gleichen chemischen Umgebungen an den Methylgruppen des Siliciums führen sollten, Inversion am Element der 5. Hauptgruppe oder intermolekularer Chloraustausch, ziehen wir den zweiten Mechanismus vor. Die Gründe hierfür sind zum einen die erfahrungsgemä $\beta$ sehr hohen Inversionsbarrieren bei diesen Elementen (siehe Lit. [13-15]) und vor allem die nachgewiesene Konzentrationsabhängigkeit (die gleichzeitige Abhängigkeit von der Temperatur ist hierbei nicht ungewöhnlich). Offenbar liegen hier ähnliche Voraussetzungen vor, wie bei einem schon länger beschriebenen AsNC-Cyclus, der am Arsenatom mit Chlor substituiert ist [7]. Wie aus den Strukturbestimmungen zu entnehmen (siehe weiter hinten), sind in 3, 4 und 5 die Element-Chlorbindungen auffällig aufgeweitet. Das Chloratom sollte deshalb einfach von einem Molekül auf das andere transferierbar sein. Beim Cyclus 5 ist die „,Destabilisierung“" der $\mathrm{Bi}-\mathrm{Cl}$-Bindung laut Struktur- 
bestimmung besonders augenfällig. Der ausgewählte Konzentrations- bzw. Temperaturbereich $(370-250 \mathrm{~K})$ genügt bei 5 nicht, um ein Aufspalten der $\mathrm{Me}_{2} \mathrm{Si}$ Signale zu beobachten, da der Austauschprozeß zu schnell verläuft.

\section{Röntgenstrukturbestimmungen an den Verbindungen $\mathrm{Me}_{2} \mathrm{Si}\left(\mathrm{N}^{\mathrm{t}} \mathrm{Bu}\right)_{2} \mathrm{El}-\mathrm{Cl}(\mathrm{El}=\mathrm{As}(3)$, Sb (4), Bi (5)) und $\mathrm{Me}_{2} \mathrm{Si}\left(\mathrm{N}^{\mathrm{t} B u}\right)_{2} \mathrm{Bi}\left(\mathrm{N}^{\mathrm{t} B u}\right) \mathrm{SiMe}_{2}\left(\mathrm{~N}^{\mathrm{t} B u}\right) \mathrm{Bi}\left(\mathrm{N}^{\mathrm{t} B u}\right)_{2} \mathrm{SiMe}_{2}$ (6)}

Die wesentlichen Daten zum Kristall und zur Strukturbestimmung von 3-6 sind in Tab. 1 zusammengefaßt. Bei allen Strukturen konnten die Lagen der Schweratome Harkersymmetrien entnommen werden, während diejenigen der leichteren Atome aus Differenz-Fourier-Synthesen folgten. Bei der Struktur von 4 und $\mathbf{5}$ sind die tert-Butylgruppen auf zwei verschiedene Lagen verteilt (Splitatomlagen). Diese Atome sowie die endständigen Methylkohlenstoffatome der tert-Butylgruppen in 6 erhielten isotrope Temperaturfaktoren, während allen

Tabelle 1 Daten zu den Kristallen und den Strukturbestimmungen von 3, 4, 5 und 6

$\mathbf{C}_{10} \mathbf{H}_{24} \mathbf{A s C l N}_{2} \mathrm{Si}(310,8 \mathrm{~g} / \mathrm{mol})(3)$, monoklin, Raumgruppe P2/ $/ \mathrm{c}, \mathrm{a}=920,0(6), \mathrm{b}=1462,9(9)$, $\mathrm{c}=1240,8(7) \mathrm{pm}, \quad \beta=105,8(1)^{\circ}, \quad \mathrm{V}=1607 \cdot 10^{6} \mathrm{pm}^{3}, \quad \mathrm{Z}=4, \quad \mathrm{D}_{\mathrm{x}}=1,28 \mathrm{~g} / \mathrm{cm}^{3}, \quad \mu(\mathrm{MoK} \alpha)=$ $22,9 \mathrm{~cm}^{-1}$, Kristallabmessungen : $1 \times 0,5 \times 0,3 \mathrm{~mm}^{3}$.

Vierkreisdiffraktometer (Stoe, Darmstadt, MoK $x$-Strahlung mit Graphitmonochromator, $\omega / 2 \Theta-$ Scan im Meßbereich $6<2 \Theta<45^{\circ} ; 2099$ Reflexe, davon 147 als, unbeobachtet" eingestuft $\left(\mathrm{F}_{0} \leq 3 \sigma_{\mathrm{F}_{0}}\right)$, LP-Korrektur; Reflex/Parameter-Verhältnis $=12,2$; Strukturbestimmung über Schweratommethode. Gewichtsschema: $\mathrm{W}=1,72 /\left(\sigma_{\mathrm{F}}^{2}+0,00133 \mathrm{~F}^{2}\right)$.

R-Wert: $\Sigma|| \mathbf{F}_{\mathrm{o}}|-| \mathbf{F}_{\mathrm{c}}|| / \Sigma\left|\mathbf{F}_{\mathrm{o}}\right|=0,040$.

$\mathbf{C}_{10} \mathbf{H}_{24} \mathbf{C l N}_{2} \mathbf{S b S i} \quad(357,6 \mathrm{~g} / \mathrm{mol}) \quad(4)$, orthorhombisch, Raumgruppe Pnma, a $=1140,2(8), \mathrm{b}=$ $1095,7(7), c=1328,7(9) \mathrm{pm}, \mathrm{V}=1660 \cdot 10^{6} \mathrm{pm}^{\mathbf{3}}, \mathrm{Z}=4, \mathrm{D}_{\mathrm{x}}=1,43 \mathrm{~g} / \mathrm{cm}^{3}, \mu(\operatorname{MoK} \alpha)=18,5 \mathrm{~cm}{ }^{-1}$, Kristallabmessungen: $0,2 \times 0,3 \times 0,7 \mathrm{~mm}^{3}$.

Vierkreisdiffraktometer (Siemens AED 2), MoK $\alpha$-Strahlung mit Graphitmonochromator, $\omega$-Scan im Meßbereich $2<2 \Theta<50^{\circ} ; 1470$ Reflexe, davon 332 als , unbeobachtet" eingestuft $\left(\mathrm{F}_{\mathrm{o}} \leq 2 \sigma_{\mathrm{F}_{0}}\right)$, LP-Korrektur, Reflex/Parameter-Verhältnis $=15,4 ;$ Strukturbestimmung über Schweratommethode. Empirische Absorptionskorrektur. Gewichtsschema: $W=0,79 /\left(\sigma_{\mathrm{F}}^{2}+0,0090 \cdot \mathrm{F}^{2}\right)$.

R.Wert: $\Sigma|| F_{0}|-| F_{0}|| / \Sigma_{0}\left|F_{0}\right|=0,052$.

$\mathbf{C}_{10} \mathbf{H}_{24} \mathbf{B i C I N} 2 \mathrm{Si}(444,8 \mathrm{~g} / \mathrm{mol})(5)$, orthorhombisch, Raumgruppe Pnma, a $=1105,1(5), \mathrm{b}=1123,3(5)$ $\mathbf{c}=1340,0(8) \mathrm{pm}, \mathrm{V}=1663 \cdot 10^{6} \mathrm{pm}^{3}, \mathrm{Z}=4, \mathrm{D}_{\mathrm{x}}=1,77 \mathrm{~g} / \mathrm{cm}^{3}, \mu(\mathrm{MoK} \alpha)=108 \mathrm{~cm}^{-1}$, Kristall. abmessungen: $0,2 \times 0,3 \times 1 \mathrm{~mm}^{3}$.

Vierkreisdiffraktometer (Stoe, Darmstadt), MoK $\alpha$-Strahlung mit Graphitmonochromator, $\omega / 2 \Theta-$ Scan im Meßbereich $5<2 \Theta<45^{\circ} ; 1537$ Reflexe, LP-Korrektur, Reflex/Parameter-Verhältnis = 20,7; Strukturbestimmung über Schweratommethode. Gewichtsschema $W=0,15 /\left(\sigma_{\mathrm{F}}^{2}+0,0583 \mathrm{~F}^{2}\right)$. R-Wert $=\Sigma|| \mathbf{F}_{0}|-| \mathbf{F}_{\mathrm{c}}|| / \Sigma\left|\mathbf{F}_{\mathrm{o}}\right|=0,058$.

$\mathbf{C}_{30} \mathbf{H}_{72} \mathbf{B i}_{2} \mathbf{N}_{6} \mathbf{S i}_{3} \cdot(1019,1 \mathrm{~g} / \mathrm{mol})(6)$, monoklin, Raumgruppe P2 $/$ c. a $=1474,2(3), \mathrm{b}=1477,6(3)$, $\mathrm{c}=1997,7(6) \mathrm{pm}, \quad \beta=94,68(8)^{\circ}, \quad \mathrm{V}=4337 \cdot 10^{6} \mathrm{pm}^{3}, \quad Z=4, \quad \mathrm{D}_{\mathrm{x}}=1,56 \mathrm{~g} / \mathrm{cm}^{3}, \quad \mu(\mathrm{MoK} \alpha)=$ $82,0 \mathrm{~cm}^{-1}$, Kristallabmessungen: $0,5 \times 0,7 \times 1,2 \mathrm{~mm}^{3}$.

Vierkreisdiffraktometer (Siemens AED2), MoKx-Strahlung mit Graphitmonochromator, $\omega$-Scan im MeBbereich $3<2 \Theta<45^{\circ} ; 5469$ Reflexe, davon 530 als , unbeboachtet" eingestuft $\left(\mathrm{F}_{0} \leq 2 \sigma_{F_{0}}\right)$, I.P-Korrektur; Reflex/Parameter-Verhältnis = 13,0; Strukturbestimmung über Schweratommethode, Empirische Absorptionskorrektur. Gewichtsschema $W=1,088 /\left(\sigma_{\mathrm{F}}^{2}+0,000677 \mathrm{~F}^{2}\right)$.

R-Wert: $\Sigma|| \mathrm{F}_{\mathrm{o}}|-| \mathrm{F}_{\mathrm{c}}|| / \Sigma\left|\mathbf{F}_{\mathrm{o}}\right|=0,052$. 
Tabelle 2 Die Ortskoordinaten und der Parameter des Debye-Waller-Faktors für die Atome der asymmetrischen Einheit von $\mathrm{Me}_{2} \mathrm{Si}\left(\mathrm{N} \mathrm{BBu}_{2} \mathrm{AsCl}(3)\right.$. Die Standardabweichungen in Klammern beziehen sich (wie bei den Tab. 3-5) auf die letzte Stelle der Zahlenwerte. Die angegebenen B-Werte des Temperaturfaktors $\exp \left(-\mathrm{B} \cdot \sin ^{2} \Theta / \lambda^{2}\right)$ wurden nach HAMILTON [17] soweit nötig aus den $\mathrm{u}_{\mathrm{ij}}$-Werten der Atome [16! berechnet

\begin{tabular}{|c|c|c|c|c|}
\hline Atoin & $x$ & $\mathrm{y}$ & $\mathbf{z}$ & $\mathbf{B}\left[10^{4}-\mathrm{pm}^{2}\right]$ \\
\hline As & $0,03903(4)$ & $0,12705(2)$ & $0,07327(3)$ & $3,09(2)$ \\
\hline$S i$ & $0,1913(3)$ & $0,2692(1)$ & $0,1827(1)$ & $3,39(4)$ \\
\hline $\mathrm{Cl}$ & $-0,0613(1)$ & $0,0553(1)$ & $0,2062(1)$ & $5,24(5)$ \\
\hline$N(1)$ & $0,0098(3)$ & $0,2484(2)$ & $0,0967(2)$ & $3,21(6)$ \\
\hline $\mathrm{N}(2)$ & $0,2316(3)$ & $0,1560(2)$ & $0,1542(2)$ & $3,22(6)$ \\
\hline$C(3)$ & $0,2990(7)$ & $0,3589(3)$ & $0,1286(5)$ & $6,2(2)$ \\
\hline$C(4)$ & $0,2042(6)$ & $0,2939(3)$ & $0,3320(3)$ & $5,6(2)$ \\
\hline$C(1)$ & $-0,1361(4)$ & $0,2979(2)$ & $0,0653(3)$ & $3,8(1)$ \\
\hline$C(11)$ & $-0,2530(5)$ & $0,2417(3)$ & $-0,0196(4)$ & $5,6(2)$ \\
\hline$(12)$ & $-0,1915(5)$ & $0,3125(3)$ & $0,1717(4)$ & $5,2(2)$ \\
\hline$C(13)$ & $-0,1121(7)$ & $0,3914(3)$ & $0,0160(5)$ & $6,5(2)$ \\
\hline$C(y)$ & $0,3596(4)$ & $0,0923(3)$ & $0,1929(3)$ & $4,1(1)$ \\
\hline$C(21)$ & $0,3293(6)$ & $0,0040(3)$ & $0,1233(5)$ & $6,6(2)$ \\
\hline$(x(22)$ & $0,3801(6)$ & $0,0677(4)$ & $0,3173(4)$ & $5,9(2)$ \\
\hline$(\times 23)$ & $0,5025(6)$ & $0,1400(4)$ & $0,1812(6)$ & $6,8(2)$ \\
\hline
\end{tabular}

Tabelle 3 Die Ortskoordinaten und der Parameter des Debye-Waller-Faktors für die Atome der asymmetrischen Einheit von $\mathrm{Me}{ }_{2} \mathrm{Si}\left(\mathrm{NtBu}_{2} \mathrm{SbCl}(4), \mathrm{C}(2), \mathrm{C}\left(2^{\prime}\right), \mathrm{C}(3),\left(3^{\prime}\right), \mathrm{C}(4), \mathrm{C}\left(4^{\prime}\right)\right.$ besetzen Lagen $z u$ 50 siehe Tab. 2

\begin{tabular}{lllll}
\hline Atom & $\mathrm{x}$ & $\mathrm{y}$ & $\mathrm{z}$ & $\mathrm{B}\left[10^{\mathbf{1} \cdot} \mathrm{pm} \mathrm{m}^{2}\right]$ \\
\hline $\mathrm{Sb}$ & $0,21290(6)$ & 0,2500 & $0,25008(4)$ & $4,21(5)$ \\
$\mathrm{Cl}$ & $0,4146(3)$ & 0,2500 & $0,3184(2)$ & $6,8(1)$ \\
$\mathrm{Si}$ & $0,2547(3)$ & 0,2500 & $0,0419(2)$ & $4,32(8)$ \\
$\mathrm{N}$ & $0,2360(5)$ & $0,1382(5)$ & $0,1332(4)$ & $4,2(2)$ \\
$\mathrm{C}(1)$ & $0,2374(7)$ & $0,0065(7)$ & $0,1323(6)$ & $5,1(3)$ \\
$\mathrm{C}(2)$ & $0,208(2)$ & $-0,048(2)$ & $0,029(1)$ & $\mathbf{1 0 , 8 ( 5 )}$ \\
$\mathrm{C}\left(2^{\prime}\right)$ & $0,319(2)$ & $-0,034(4)$ & $0,044(2)$ & $10,8(5)$ \\
$\mathrm{C}(3)$ & $0,151(1)$ & $-0,049(2)$ & $0,209(1)$ & $8,9(4)$ \\
$\mathrm{C}\left(3^{\prime}\right)$ & $0,117(2)$ & $-0,044(3)$ & $0,099(2)$ & $8,1(3)$ \\
$\mathrm{C}(4)$ & $0,359(1)$ & $-0,042(2)$ & $0,161(1)$ & $8,1(3)$ \\
$\mathrm{C}\left(4^{\prime}\right)$ & $0,280(3)$ & $-0,067(4)$ & $0,223(2)$ & $8,9(4)$ \\
$\mathrm{C}(5)$ & $0,142(1)$ & 0,2500 & $-0,058(1)$ & $7,5(8)$ \\
$\mathrm{C}(6)$ & $0,408(1)$ & 0,2500 & $-0,014(1)$ & $9,2(9)$ \\
\hline
\end{tabular}

Tabelle 4 Die Ortskoordinaten und der Parameter des Debye-Waller-Faktors für die Atome der asymmetrischen Einheit von $\mathrm{Me}_{2} \mathrm{Si}(\mathrm{NtBu})_{2} \mathrm{BiCl}(5)$. $\mathrm{C}(2), \mathrm{C}\left(2^{\prime}\right), \mathrm{C}(3), \mathrm{C}\left(3^{\prime}\right), \mathrm{C}(4), \mathrm{C}^{\prime}\left(4^{\prime}\right)$ besetzten Lagen zu $50_{\%}^{\circ}$. Weitere Erläuterungen siehe Tab. 2

\begin{tabular}{|c|c|c|c|c|}
\hline Atom & $\mathbf{x}$ & $\mathrm{y}$ & $\mathbf{z}$ & $\mathrm{B}\left[10^{4} \cdot \mathrm{pm}^{2}\right]$ \\
\hline $\mathrm{Bi}$ & $0,19257(4)$ & 0,2500 & $0,23609(5)$ & $3,22(4)$ \\
\hline (!) & $0,4233(4)$ & 0,2500 & $0,3127(4)$ & $5,2(2)$ \\
\hline $\mathrm{Si}$ & $0,2530(4)$ & 0,2500 & $0,0241(3)$ & $4,1(2)$ \\
\hline $\mathrm{N}$ & $0,2257(7)$ & $0,1391(7)$ & $0,1106(7)$ & $4,1(2)$ \\
\hline$C(1)$ & $0,237(1)$ & $0,0064(8)$ & $0,115(1)$ & $5,1(5)$ \\
\hline$C(2)$ & $0,206(4)$ & $-0,0049(4)$ & $0,007(5)$ & $10(1)$ \\
\hline$C\left(2^{\prime}\right)$ & $0,270(4)$ & $-0,041(3)$ & $0,005(3)$ & $7,2(6)$ \\
\hline$C(3)$ & $0,143(2)$ & $-0,044(2)$ & $0,192(2)$ & $3,8(3)$ \\
\hline$C\left(3^{\prime}\right)$ & $0,120(4)$ & $-0,038(4)$ & $0,144(4)$ & $10(1)$ \\
\hline$C(1)$ & $0,372(3)$ & $-0,019(3)$ & $0,149(3)$ & $6,3(7)$ \\
\hline$C\left(4^{\prime}\right)$ & $0,351(4)$ & $-0,042(4)$ & $0,174(3)$ & $6,7(8)$ \\
\hline$C(5)$ & $0,142(4)$ & 0,2500 & $-0,081(2)$ & $10(2)$ \\
\hline$(\because 6)$ & $0,423(2)$ & 0,2500 & $-0,021(3)$ & $9(1)$ \\
\hline
\end{tabular}


Takelle 5 Die Ortskoordinaten und der Parameter des Debye-Waller-Faktors für die Atom: der asymuetrischen Ein-

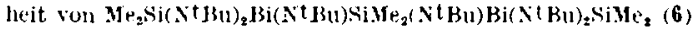

\begin{tabular}{|c|c|c|c|c|}
\hline$A t o n$ & $\mathrm{x}$ & $y$ & $z$ & $H\left[10^{\prime} \cdot \mathrm{pm}^{*}\right]$ \\
\hline $\mathbf{J i}(1)$ & $0.13700(3)$ & $0,13843(3)$ & $0.01399(2)$ & $3.61 .(2)$ \\
\hline $\operatorname{Bi} \mathbf{i}(2)$ & $0,3606.4(3)$ & $0.84341(3)$ & $0,85019(2)$ & $3.43(2)$ \\
\hline $\operatorname{si}(1)$ & $0,2532(2)$ & $11,9715(3)$ & $0,9530(2)$ & $3.9(1)$ \\
\hline $\operatorname{sil}(2)$ & $0,3268(2)$ & $0,7019(2)$ & $0,7189(2)$ & $4.4(1)$ \\
\hline $\mathrm{Si}(3)$ & $0,1580(3)$ & $0.326 .4(2)$ & $0,0580(2)$ & $5.3(2)$ \\
\hline$X(1)$ & $0,2462(3)$ & $0,0885(5)$ & $0,95.18(4)$ & $3,5(4)$ \\
\hline$X(2)$ & $0,2390(6)$ & $01,9195(5)$ & $0,8750(5)$ & $3,3(4)$ \\
\hline$X(3)$ & $(0,3523(6)$ & $0.8142(6)$ & $0,7429(4)$ & $3,9(4)$ \\
\hline$X(t)$ & $0,3151(7)$ & $0,7050(6)$ & $0,8338(5)$ & $1.6(5)$ \\
\hline$N(5)$ & $0.1044(6)$ & $0,2775(5)$ & $0,9884(5)$ & $4,6(5)$ \\
\hline$X(b)$ & $0,2043(7)$ & $0.226+4(6)$ & $0,0879(4)$ & $1,7(5)$ \\
\hline$(1)$ & $0.078(1)$ & $0,391(1)$ & $0,1147(8)$ & $9(1)$ \\
\hline$I^{\prime}(2)$ & $0.2+4(1)$ & $0,4133(9)$ & $0,0397(9)$ & $9(1)$ \\
\hline$C(3)$ & $0.246(1)$ & $0,19833(8)$ & $0.1544(0)$ & $5,5(7)$ \\
\hline$(31)$ & $0,249(1)$ & $0,112(1)$ & $0,1488(8)$ & $7,4(4)$ \\
\hline$c(32)$ & $0,174(1)$ & $0,181(1)$ & $0,203(1)$ & $9,9(5)$ \\
\hline$C^{\prime}(33)$ & $0,304(1)$ & $0,272(1)$ & $0,18.45(8)$ & $7.5(4)$ \\
\hline$r^{\prime}(1)$ & $0,0361(9)$ & $0,3086(8)$ & $0,9334(\pi)$ & $5,4(7)$ \\
\hline$((11)$ & $0,073(1)$ & $0,3927(9)$ & $0,8996(8)$ & $6,6(3)$ \\
\hline$(42)$ & $0,017(1)$ & $0,235(1)$ & $0.8819(8)$ & $7,0(4)$ \\
\hline$C(43)$ & $-0,055(1)$ & $0,33:(1)$ & $0,9623(9)$ & $7,6(4)$ \\
\hline$C(5)$ & $0,3651(8)$ & $0,9313(9)$ & $0.9981(\hat{0})$ & $5.7(7)$ \\
\hline$f^{\prime}\left(t^{i}\right)$ & $0,170(1)$ & $0,9209(7)$ & $0,0124(7)$ & $5.6(7)$ \\
\hline ('(z) & $0,3159(8)$ & $0,1.494(7)$ & $0.9273(6)$ & $4.2(5)$ \\
\hline$c(8)$ & $0.1502(8)$ & $0,918 \vec{i}(8)$ & $0.8329(6)$ & $4,7(6)$ \\
\hline C(71) & $0,2730(3)$ & $0,2297(9)$ & $0,8918(7)$ & $5.9(3)$ \\
\hline$(72)$ & $0,372(1)$ & $0,100(1)$ & $0,8772(\bar{i})$ & $6,5(3)$ \\
\hline$(:(73)$ & $0,384(1)$ & $0,182(1)$ & $0,9843(8)$ & $7,9(3)$ \\
\hline$c(81)$ & $(1,1) 82(1)$ & $0,988(1)$ & $0,8530(9)$ & $8.0(4)$ \\
\hline$C(82)$ & $0,106(1)$ & $0,827(1)$ & $0,837(1)$ & $9, f(5)$ \\
\hline$(83)$ & $0,163(1)$ & $0,932(1)$ & $0,7583(8)$ & $8,6(4)$ \\
\hline$\left(C^{\prime}(1)\right.$ & $0,220(1)$ & $0,666(1)$ & $0,6979(7)$ & $6,8(8)$ \\
\hline$C(12)$ & $0,417(1)$ & $0.6219(9)$ & $0, \pi 218(8)$ & $6,4(8)$ \\
\hline$(2(10)$ & $0,311(1)$ & $0,6371(\beta)$ & $0,8849(8)$ & $6,0(7)$ \\
\hline$C(9)$ & $0,3960(9)$ & $0,8075(8)$ & $0,6946(\overline{7})$ & $5,2(6)$ \\
\hline$C(91)$ & $0,353(1)$ & $0.8 .48(1)$ & $0,6228(9)$ & $9,3(5)$ \\
\hline$c(92)$ & $0,497(1)$ & $0,849(1)$ & $0,694(1)$ & $9,0(5)$ \\
\hline$c(93)$ & $0,388(1)$ & $0.9693(8)$ & $0.7069(8)$ & $7,0(3)$ \\
\hline c(101) & $0,260(1)$ & $0,670(1)$ & $0,9435(8)$ & $7,7(1)$ \\
\hline$C^{\prime}(102)$ & $0,405(1)$ & $0,610(1)$ & $0,913(1)$ & $11,2(6)$ \\
\hline$(9103)$ & $0,267(2)$ & $0,5,53(1)$ & $0,858(1)$ & $12,2(6)$ \\
\hline
\end{tabular}

anderen Atomen anisotrope Temperaturfaktoren zugeordnet wurden. In den Tab. 2 bis $\overline{\mathbf{5}}$ sind die Lageparameter und isotropen Temperaturfaktoren der $\mathrm{A}$ tome in den Verbindungen aufgeführt. Die Tabellen der anisotropen Temperaturfaktoren und die $\mathbf{F}_{\mathrm{u}}-\mathrm{F}_{\mathrm{v}}$-Tabellen für alle vier Verbindungen sind dokumentiert [16].

Diskussion der Strukturen von $\mathrm{Me}_{2} \mathrm{Si}\left(\mathrm{N}^{t} \mathrm{Bu}\right)_{2} \mathrm{El}-\mathrm{Cl}(\mathrm{El}=\mathrm{A}(3), \mathrm{Sb}(4)$, $\operatorname{Bi}(\mathbf{5}))$

Als Ergebnis der Strukturbestimmung ist in Abb. 1 ein ,,isoliertes" Molekül 3 und zwei über das Inversionszentrum,,verknüpfte" Einheiten dargestellt. Als Beispiel für die Strukturen der Verbindungen 4 und $\mathbf{5}$, die isotyp zueinander sind, ist ein Ausschnitt aus der Kristallstruktur von 5 in Abb. 2 gezeigt. Ein 

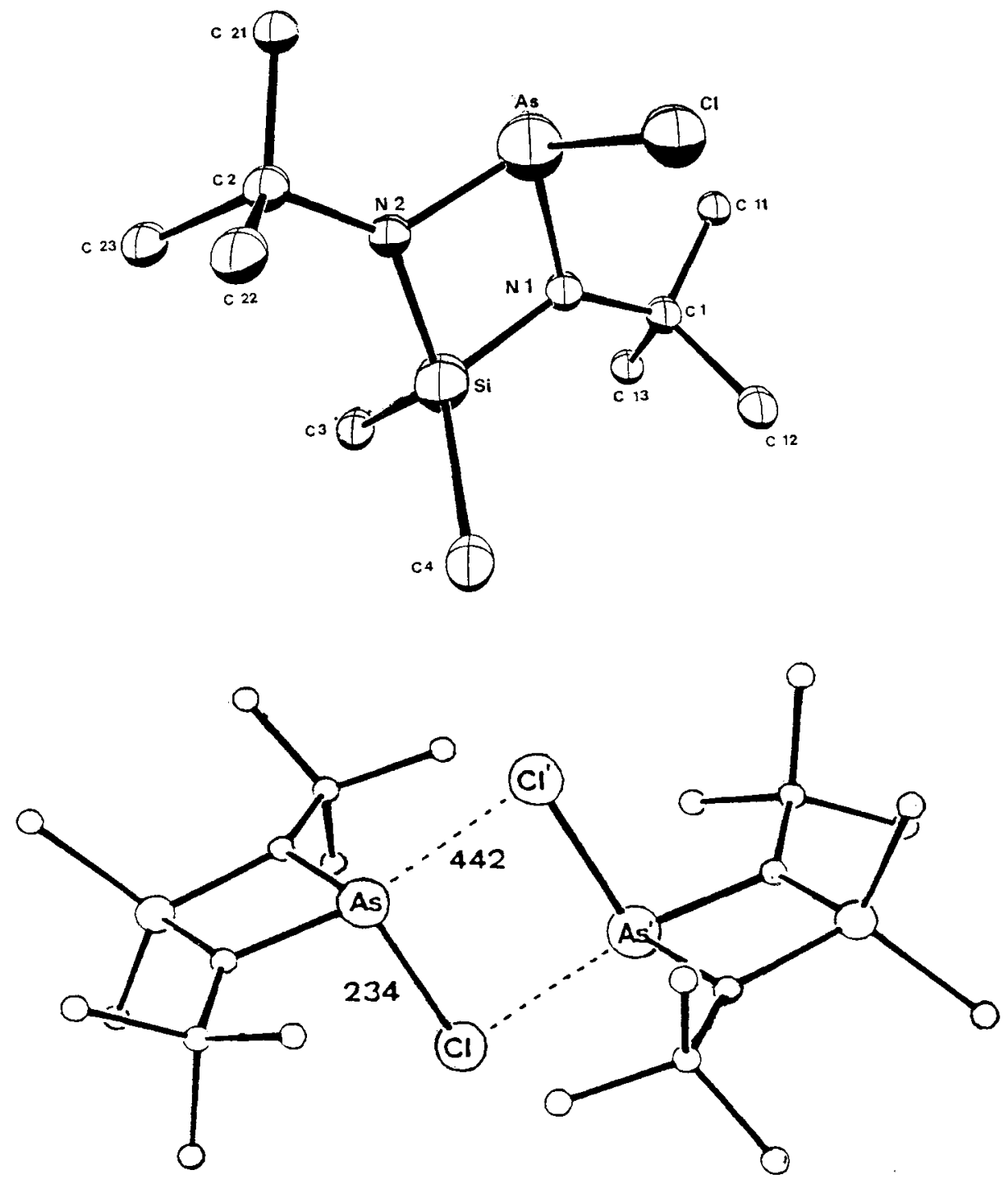

Abb. 1 Zeichnerische Darstellung eines ,isolierten“ Moleküls $\mathrm{Me}_{2} \mathrm{Si}\left(\mathrm{N}^{\mathrm{t}} \mathrm{Bu}\right)_{2} \mathrm{AsCl}$ (3) mit Atombezeichnungen und eines zentrosymmetrischen ,Dimeren“.

Vergleich beider Figuren macht deutlich, daß sich die Kristallstruktur des Arsenderivates deutlich von den anderen beiden Derivaten unterscheidet. Bei letzteren Verbindungen ist eine eindimensionale, an den Chloratomen leicht geknickte $\mathrm{El}-\mathrm{Cl} \cdots \mathrm{El}-\mathrm{Cl} \cdots$-Kette das bestimende Strukturelement; bei $\mathbf{3}$ liegen $\mathrm{zu}$ Dimeren assoziierte Moleküle vor. Die As -Cl-Abstände innerhalb der Moleküle sind mit 234,5(1) pm jedoch wesentlich kürzer als die zwischen den Molekülen paarweise auftretenden As ..Cl-Kontakte von 442 pm. Die „Dimerisierung“ bei 3 muß daher als eine van-der-Waals-Wechselwirkung gedeutet werden. Bei 4 und 


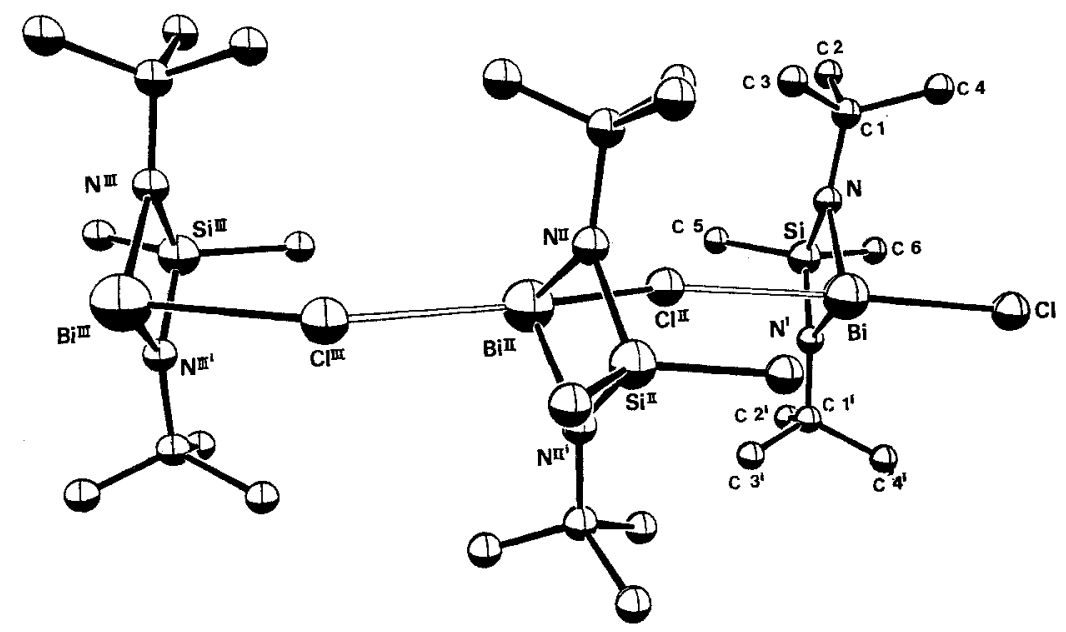

Abb. 2 Ausschnitt aus der Kristallstruktur von $\mathrm{Me}_{2} \mathrm{Si}\left(\mathrm{N}^{\mathrm{t}} \mathrm{Bu}\right)_{2} \mathrm{BiCl}(5)$ mit Atombenennungen. Die mit i bezeichneten Atome gehen aus den unbezeichneten durch die Spiegelebene hervor. Das Molekül II entsteht über eine Gleitoperation, das Molekül III über Translation.

Tabelle 6 Bindungslängen (pm) und Winkel $\left(^{\circ}\right)$ im Molekül $\mathrm{Me}_{2} \mathrm{Si}\left(\mathrm{N}^{t} \mathrm{Bu}\right)_{2} \mathrm{AsCl}(\mathbf{3})$. Bei den Standardabweichungen in Klammern wurden die Ungenauigkeiten in den Ortskoordinaten berücksichtigt

$\begin{array}{lrlrlll}\mathrm{As}-\mathrm{Cl} & 234,5(1) & \mathrm{Cl}-\mathrm{As} \cdots \mathrm{Si} & 104,1(0) & & \\ \mathrm{As}-\mathrm{N}(1) & 183,1(3) & \mathrm{N}(1)-\mathrm{As}-\mathrm{Cl} & 102,7(1) & & \\ \mathrm{As}-\mathrm{N}(2) & 183,3(3) & \mathrm{N}(2)-\mathrm{As}-\mathrm{Cl} & 103,0(1) & & \\ & & \mathrm{N}(2)-\mathrm{As}-\mathrm{N}(1) & 81,4(1) & & \\ \mathrm{Si}-\mathrm{N}(1) & 174,6(3) & & & & \\ \mathrm{Si}-\mathrm{N}(2) & 175,3(3) & \mathrm{N}(2)-\mathrm{Si}-\mathrm{N}(1) & 86,1(1) & \mathrm{C}(11)-\mathrm{C}(1)-\mathrm{N}(1) & 109,7(3) \\ \mathrm{Si}-\mathrm{C}(3) & 187,6(4) & \mathrm{C}(3)-\mathrm{Si}-\mathrm{N}(1) & 114,4(2) & \mathrm{C}(12)-\mathrm{C}(1)-\mathrm{N}(1) & 109,1(3) \\ \mathrm{Si}-\mathrm{C}(4) & 185,9(4) & \mathrm{C}(3)-\mathrm{Si}-\mathrm{N}(2) & 115,2(2) & \mathrm{C}(12)-\mathrm{C}(1)-\mathrm{C}(11) & 109,2(3) \\ & & \mathrm{C}(4)-\mathrm{Si}-\mathrm{N}(1) & 115,7(2) & \mathrm{C}(13)-\mathrm{C}(1)-\mathrm{N}(1) & 108,7(3) \\ \mathrm{N}(1)-\mathrm{C}(1) & 148,1(5) & \mathrm{C}(4)-\mathrm{Si}-\mathrm{N}(2) & 115,2(2) & \mathrm{C}(13)-\mathrm{C}(1)-\mathrm{C}(11) & 110,8(4) \\ \mathrm{N}(2)-\mathrm{C}(2) & 147,6(5) & \mathrm{C}(4)-\mathrm{Si}-\mathrm{C}(3) & 108,9(2) & \mathrm{C}(13)-\mathrm{C}(1)-\mathrm{C}(12) & 109,3(3) \\ & & & & & \\ \mathrm{C}(1)-\mathrm{C}(11) & 152,5(6) & \mathrm{C}(1)-\mathrm{N}(1)-\mathrm{As} & 126,4(2) & \mathrm{C}(21)-\mathrm{C}(2)-\mathrm{N}(2) & 109,9(3) \\ \mathrm{C}(1)-\mathrm{C}(12) & 155,5(6) & \mathrm{C}(1)-\mathrm{N}(1)-\mathrm{Si} & 136,4(2) & \mathrm{C}(22)-\mathrm{C}(2)-\mathrm{N}(2) & 110,0(3) \\ \mathrm{C}(1)-\mathrm{C}(13) & 153,8(5) & \mathrm{Si}-\mathrm{N}(1)-\mathrm{As} & 96,2(1) & \mathrm{C}(22)-\mathrm{C}(2)-\mathrm{C}(21) & 108,6(4) \\ & & & \mathrm{C}(23)-\mathrm{C}(2)-\mathrm{N}(2) & 108,2(3) \\ \mathrm{C}(2)-\mathrm{C}(21) & 153,6(6) & \mathrm{C}(2)-\mathrm{N}(2)-\mathrm{As} & 126,8(2) & \mathrm{C}(23)-\mathrm{C}(2)-\mathrm{C}(21) & 111,1(4) \\ \mathrm{C}(2)-\mathrm{C}(22) & 154,6(6) & \mathrm{C}(2)-\mathrm{N}(2)-\mathrm{Si} & 136,4(2) & \mathrm{C}(23)-\mathrm{C}(2)-\mathrm{C}(22) & 109,0(3) \\ \mathrm{C}(2)-\mathrm{C}(23) & 153,0(6) & \mathrm{Si}-\mathrm{N}(2)-\mathrm{As} & 95,9(1) & & \end{array}$

Abstände zwischen nichtbindenden Atomen
As $\cdots$ Si
$266,3(1)$
$\mathrm{N}(1) \cdots \mathrm{N}(2)$
$238,9(2)$
As $\cdots \mathrm{Cl}(1) \quad 442,3(1)$ 
Tabelle 7 Bindungslängen $(\mathrm{pm})$ und Winkel $\left({ }^{\circ}\right)$ im Molekül $\mathrm{Me}_{2} \mathrm{Si}\left(\mathrm{N}^{\mathrm{t}} \mathrm{Bu}\right)_{2} \mathrm{SbCl}(\mathbf{4})$. Standardabweichungen wie bei Tab. 6

\begin{tabular}{|c|c|c|c|c|c|}
\hline $\mathrm{Cl}-\mathrm{Sb}$ & $247,2(3)$ & $\mathrm{Si} \cdots \mathrm{Sb}-\mathrm{Cl}$ & $101,8(1)$ & $C(2)-C(1)-N$ & $113(1)$ \\
\hline $\mathrm{N}-\mathrm{Sb}$ & $199,5(6)$ & $\mathrm{N}-\mathrm{Sb}-\mathrm{Cl}$ & $99,4(2)$ & $\mathrm{C}(3)-\mathrm{C}(1)-\mathrm{N}$ & $112(1)$ \\
\hline $\mathrm{N}-\mathrm{Si}$ & $173,7(6)$ & $\mathrm{N}-\mathrm{Sb}-\mathrm{N}$ & $75,7(3)$ & $\mathrm{C}(4)-\mathrm{C}(1)-\mathrm{N}$ & $111(1)$ \\
\hline $\mathrm{C}(6)-\mathrm{Si}$ & $189(1)$ & & & $C(2)-C(1)-C(3)$ & $107(1)$ \\
\hline \multirow[t]{4}{*}{$\mathrm{C}(5)-\mathrm{Si}$} & $185(1)$ & $\mathrm{C}(6)-\mathrm{Si}-\mathrm{N}$ & $112,7(4)$ & $C(2)-C(1)-C(4)$ & $107(1)$ \\
\hline & & $\mathrm{N}-\mathrm{Si}-\mathrm{N}^{\prime}$ & $89,7(3)$ & $C(3)-C(1)-C(4)$ & $105,9(9)$ \\
\hline & & $\mathrm{C}(5)-\mathrm{Si}-\mathrm{N}^{\prime}$ & $114,6(4)$ & & \\
\hline & & $C(5)-S i-C(6)$ & $111,0(8)$ & & \\
\hline \multirow[t]{2}{*}{$C(1)-N$} & $144(1)$ & & & & \\
\hline & & & & $\mathrm{C}\left(2^{\prime}\right)-\mathrm{C}(1)-\mathrm{N}$ & $107(2)$ \\
\hline$C(2)-C(1)$ & $153,9(9)$ & & & $\mathrm{C}\left(3^{\prime}\right)-\mathrm{C}(1)-\mathrm{N}$ & $110(1)$ \\
\hline$C(3)-C(1)$ & $154,2(9)$ & & & $\mathrm{C}\left(4^{\prime}\right)-\mathrm{C}(1)-\mathrm{N}$ & $122(2)$ \\
\hline \multirow[t]{2}{*}{$C(4)-C(1)$} & $153,2(9)$ & $\mathrm{C}(\mathbf{1})-\mathbf{N}-\mathrm{Sb}$ & $128,5(5)$ & $C\left(2^{\prime}\right)-C(1)-C\left(3^{\prime}\right)$ & $102(1)$ \\
\hline & & $\mathrm{C}(1)-\mathrm{N}-\mathrm{Si}$ & $134,2(5)$ & $\mathrm{C}\left(2^{\prime}\right)-\mathrm{C}(1)-\mathrm{C}\left(4^{\prime}\right)$ & $105(1)$ \\
\hline $\mathrm{C}\left(2^{\prime}\right)-\mathrm{C}(1)$ & $156,2(9)$ & $\mathrm{Si}-\mathrm{N}-\mathrm{Sb}$ & $97,3(3)$ & $\mathrm{C}\left(3^{\prime}\right)-\mathrm{C}(1)-\mathrm{C}\left(4^{\prime}\right)$ & $109(1)$ \\
\hline $\mathrm{C}\left(3^{\prime}\right)-\mathrm{C}(1)$ & $154(1)$ & & & & \\
\hline$C\left(4^{\prime}\right)-C(1)$ & $153(1)$ & & & & \\
\hline \multicolumn{6}{|c|}{ Weitere Abstände } \\
\hline $\mathrm{Si} \cdots \mathrm{Sb}$ & $280,6(3)$ & & & & \\
\hline $\mathrm{Sb} \cdots \mathrm{Cl}$ & $352,1(5)$ & & & & \\
\hline
\end{tabular}

Tabelle 8 Bindungslängen (pm) und Winkel $\left({ }^{\circ}\right)$ im Molekül $\mathrm{Me}_{2} \mathrm{Si}\left(\mathrm{N}^{\mathrm{t}} \mathrm{Bu}\right)_{2} \mathrm{BiCl}(5)$. Standardabweichungen wie bei Tab. 6

\begin{tabular}{llllll}
$\mathrm{Bi}-\mathrm{Cl}$ & $274,8(4)$ & $\mathrm{Si} \cdots \mathrm{Bi}-\mathrm{Cl}$ & $98,7(1)$ & $\mathrm{C}(2)-\mathrm{C}(1)-\mathrm{N}$ & $109(2)$ \\
$\mathrm{Bi}-\mathrm{N}$ & $212,4(9)$ & $\mathrm{N}-\mathrm{Bi}-\mathrm{Cl}$ & $97,8(3)$ & $\mathrm{C}(3)-\mathrm{C}(1)-\mathrm{N}$ & $109(1)$ \\
& & $\mathrm{N}-\mathrm{Bi}-\mathrm{N}^{\prime}$ & $71,8(4)$ & $\mathrm{C}(4)-\mathrm{C}(1)-\mathrm{N}$ & $106(1)$ \\
$\mathrm{Si}-\mathrm{N}$ & $172,8(8)$ & & & & \\
$\mathrm{Si}-\mathrm{C}(5)$ & $187(3)$ & $\mathrm{N}-\mathrm{Si}-\mathrm{N}^{\prime}$ & $92,2(4)$ & $\mathrm{C}\left(2^{\prime}\right)-\mathrm{C}(1)-\mathrm{N}$ & $108(2)$ \\
$\mathrm{Si}-\mathrm{C}(6)$ & $197(2)$ & $\mathrm{C}(5)-\mathrm{Si}-\mathrm{N}$ & $112,9(9)$ & $\mathrm{C}\left(3^{\prime}\right)-\mathrm{C}(1)-\mathrm{N}$ & $106(2)$ \\
& & $\mathrm{C}(6)-\mathrm{Si}-\mathrm{N}$ & $111,9(8)$ & $\mathrm{C}\left(4^{\prime}\right)-\mathrm{C}(1)-\mathrm{N}$ & $116(2)$ \\
$\mathrm{C}(1)-\mathrm{N}$ & $150(1)$ & $\mathrm{C}(6)-\mathrm{Si}-\mathrm{C}(5)$ & $113(2)$ & & \\
$\mathrm{C}(2)-\mathrm{C}(1)$ & $162(6)$ & & & $\mathrm{C}(2)-\mathrm{C}(1)-\mathrm{C}(3)$ & $108(2)$ \\
$\mathrm{C}(3)-\mathrm{C}(1)$ & $157(3)$ & & & $\mathrm{C}(2)-\mathrm{C}(1)-\mathrm{C}(4)$ & $112(2)$ \\
$\mathrm{C}(4)-\mathrm{C}(1)$ & $158(4)$ & $\mathrm{Si}-\mathrm{N}-\mathrm{Bi}$ & $98,0(4)$ & $\mathrm{C}(1)-\mathrm{C}(4)$ & $112(2)$ \\
$\mathrm{C}\left(2^{\prime}\right)-\mathrm{C}(1)$ & $161(4)$ & $\mathrm{C}(1)-\mathrm{N}-\mathrm{Bi}$ & $124,5(8)$ & $\mathrm{C}\left(2^{\prime}\right)-\mathrm{C}(1)-\mathrm{C}\left(3^{\prime}\right)$ & $109(3)$ \\
$\mathrm{C}\left(3^{\prime}\right)-\mathrm{C}(1)$ & $144(4)$ & $\mathrm{C}(1)-\mathrm{N}-\mathrm{Si}$ & $136,9(9)$ & $\mathrm{C}\left(3^{\prime}\right)-\mathrm{C}(1)-\mathrm{C}\left(4^{\prime}\right)$ & $118(3)$ \\
$\mathrm{C}\left(4^{\prime}\right)-\mathrm{C}(1)$ & $158(4)$ & & $\mathrm{C}\left(4^{\prime}\right)-\mathrm{C}(1)-\mathrm{C}\left(2^{\prime}\right)$ & $99(2)$ \\
& & & & \\
Weitere Abstände & & & & \\
$\mathrm{Bi} \cdots \mathrm{Si}$ & $291,8(5)$ & $\mathrm{N} \cdots \mathrm{N}^{\prime}$ & 249,1 & & \\
$\mathrm{Bi} \cdots \mathrm{Cl}$ & $304,7(6)$ & $\mathrm{Bi} \cdots \mathrm{Bi}$ & 553,8 & & \\
\hline
\end{tabular}


handelt es sich hingegen um stark gerichtete Bindungen zwischen den Molekülen, was sich aus dem Vergleich von intra- zu intermolekularem El-Cl-Abstand ablesen läßt $(\mathrm{Sb}-\mathrm{Cl}=247,2(3), \quad \mathrm{Sb} \cdots \mathrm{Cl}=352,1(8) ; \quad \mathrm{Bi}-\mathrm{Cl}=274,8(4)$; $\mathrm{Bi} \cdots \mathrm{Cl}=304,7(4) \mathrm{pm})$. Weitere wichtige Abstände und Winkel der Moleküle 3, 4 und 5 sind in den Tab. 6-8 zusammengestellt.

Die Elementchlorbindungen sind bei allen Molekülen nahezu senkrecht zu den planaren $\mathrm{SiN}_{2}$ El-Ringen ausgerichtet, wobei bei 4 und 5 eine Spiegelebene der Raumgruppe das Molekül in zwei Hälften teilt, während bei 3 die Abweichung von einer solchen $\mathrm{m}\left(\mathrm{C}_{\mathrm{s}}\right)$-Symmetrie nur unbedeutend ist (vgl. Tab. 6). Die Assoziation der Moleküle über Chlor-Elementbrücken nimmt von As über Sb zum Bi zu (siehe vorherigen Abschnitt!). Zwei Bindungsmodelle lassen sich diskutieren: einerseits eine Valenzaufweitung am Element der 5. Hauptgruppe ( $\mathrm{sp}^{3} \mathrm{~d}-\mathrm{Hy}$ bridisierung) und somit Säure-Base-Wechselwirkung zwischen $\mathrm{Sb}$ bzw. Bi als Acceptor und Chlor als Donor, und andererseits eine Ablösung des Chloratoms als $\mathrm{Cl}^{-}$im Festkörper und damit elektrostatische Wechselwirkung zwischen

$\mathrm{Me}_{2} \mathrm{Si}\left(\mathrm{N}^{t} \mathrm{Bu}\right)_{2} \mathrm{El}^{+}-\mathrm{Kationen}$ und dazwischen gelagerten Chlorid-Anionen. Das zweite Modell würde allerdings gleiche Abstände $\mathrm{El}-\mathrm{Cl}$ und $\mathrm{El}$...Cl voraussetzen, was offensichtlich nicht der Fall ist.

Ein Vergleich der Element-Chlorabstände mit der Summe der Kovalenzradien bzw. mit den Bindungslängen in den Elementtrichloriden (Tab. 9) zeigt, daß alle Abstände zu lang ausfallen. Die Einbindung der Elemente der 5. Hauptgruppe in die $\mathrm{SiN}_{2} \mathrm{El}$-Vierringe hat demnach eine destabilisierende Wirkung auf die Element-Chlorbindung (vgl. ${ }^{1} \mathrm{H}-\mathrm{NMR}$ ). Chemisch sollten sich die Chlorsubstituenten leicht abspalten lassen, was sich einfach nachweisen läßt [4]. Die Element-Stickstoff-Bindungslängen sind in einem Bereich, in dem man sie er-

Tabelle 9 Vergleich der El-Cl-Abstände (pm) und der El $\cdots \mathrm{CI}^{\prime}$-Abstände, die aus intermolekularen Kontakten hervorgehen, mit aus Kovalenzradien berechneten $\mathrm{Abständen} \mathrm{El}-\mathrm{Cl}_{\mathrm{ber}}$ [21] und mit den $\mathrm{El}-\mathrm{Cl}$-Abständen in den Trichloriden $\mathrm{ElCl}_{3}\left(\mathrm{El}-\mathrm{Cl}_{\text {tri }}\right)[23]$

\begin{tabular}{llllll}
\hline Verbindung & $\mathrm{El}-\mathrm{Cl}$ & $\mathrm{El} \cdots \mathrm{Cl}^{\prime}$ & $\mathrm{El}-\mathrm{Cl}_{\text {ber. }}$ & El-Cl $\mathrm{Cl}_{\text {tri }}$ \\
\hline $\mathrm{Me}_{2} \mathrm{Si}\left(\mathrm{N}^{\mathrm{t}} \mathrm{Bu}\right)_{2} \mathrm{AsCl}$ & $(\mathbf{3})$ & 234,5 & 442,3 & 219 & 217 \\
$\mathrm{Me}_{2} \mathrm{Si}\left(\mathrm{N}^{\mathrm{t}} \mathrm{Bu}\right)_{2} \mathrm{SbCl}$ & $(\mathbf{4})$ & 247,2 & 352,1 & 239 & 236 \\
$\mathrm{Me}_{2} \mathrm{Si}\left(\mathrm{N}^{\mathrm{t}} \mathrm{Bu}\right)_{2} \mathrm{BiCl}$ & $(\mathbf{5})$ & 274,8 & 304,7 & 245 & 250 \\
\hline
\end{tabular}

Tabelle 10 Vergleich der wichtigsten Abstände (pm) und Winkel $\left(^{\circ}\right)$ in den Vierringen der Verbindungen 3,4 und 5

\begin{tabular}{lrrr}
\hline & $\mathbf{3}(\mathbf{E l}=\mathrm{As})$ & $\mathbf{4}(\mathrm{El}=\mathrm{Sb})$ & $\mathbf{5}(\mathrm{El}=\mathrm{Bi})$ \\
\hline $\mathrm{El}-\mathrm{N}$ & 183,2 & 199,5 & 212,4 \\
$\mathrm{Si}-\mathrm{N}$ & 174,9 & 173,7 & 172,8 \\
$\mathrm{~N}-\mathrm{El}-\mathrm{N}$ & 81,4 & 75,7 & 71,8 \\
$\mathrm{~N}-\mathrm{Si}-\mathrm{N}$ & 86,1 & 89,7 & 92,2 \\
$\mathrm{Si} \cdots \mathrm{EI}-\mathrm{Cl}$ & 104,1 & 101,8 & 98,7 \\
\hline
\end{tabular}


wartet, [18-21], die Silicium-Stickstoff-Abstände sind relativ kurz [22] und nehmen in der Reihe 3, 4, 5 stetig ab. Die Hauptlast der Ringspannung tragen erwartungsgemäß die Schweratome $\mathrm{As}, \mathrm{Sb}$ und $\mathrm{Bi}$, an denen die spitzesten Winkel anzutreffen sind (Tab. 6-8).

In Tab. 10 sind die vergleichbaren strukturellen Parameter innerhalb 3, 4 und 5 zusammengestellt. Wie man erkennt, korrelieren zunehmende $\mathrm{El}-\mathrm{N}$ - $\mathrm{Ab}$ stände mit kleiner werdendem $\mathrm{N}-\mathrm{El}-\mathrm{N}$-Winkel, abnehmende $\mathrm{Si}-\mathrm{N}$-Abstände mit zunehmendem $\mathrm{N}-\mathrm{Si}-\mathrm{N}$-Winkel und zunehmende $\mathrm{El}-\mathrm{N}$ - mit abnehmenden $\mathrm{Si}-\mathrm{N}$-Werten. Besonders interessant ist derWinkel des $\mathrm{El}-\mathrm{Cl}$-Vektors zu der den Vierring halbierenden El $\cdots \mathrm{Si}$-Geraden $(\mathrm{Si} \cdots \mathrm{El}-\mathrm{Cl})$ : Die stetige Abnahme könnte mit einer abnehmenden Hybridisierungsneigung des schwereren Elementes interpretiert werden; wobei offenbar beim Bismutatom ein Bindungszustand erreicht wird, bei dem das nichtbindende Elektronenpaar weitgehend in einem s-Orbital lokalisiert sein sollte.

Diskussion der Struktur von

$\mathrm{Me}_{2} \mathrm{Si}\left(\mathrm{N}^{\mathrm{t}} \mathrm{Bu}\right)_{2} \mathrm{Bi}-\mathrm{N}\left({ }^{\mathrm{t}} \mathrm{Bu}\right) \mathrm{SiMe}_{2} \mathrm{~N}\left({ }^{\mathrm{t}} \mathrm{Bu}\right)-\mathrm{Bi}\left(\mathrm{N}^{\mathrm{t}} \mathrm{Bu}\right)_{2} \mathrm{SiMe}_{2}(6)$

In der Elementarzelle von 6 befinden sich 4 Moleküle auf allgemeinen Lagen, wobei die Moleküle untereinander ausschließlich über van-der-Waals-Kontakte zusammengehalten werden. In Abb. 3 ist ein Molekül 6 dargestellt und Bindungsabstände und -winkel sind in Tab. 11 enthalten. Bei Betrachtung der zeichnerischen Darstellung erkennt man, daß eine pseudo-zweizählige Achse durch das Atom Si(1) etwa senkrecht zur gewählten Abbildungsebene verläuft. Jedes Bismutatom ist in einen $\mathrm{SiN}_{2} \mathrm{Bi}$-Vierring eingebaut und betätigt eine dritte Valenz zu einem Stickstoffatom eines $\mathrm{Me}_{2} \mathrm{Si}\left(\mathrm{N}^{\mathrm{t}} \mathrm{Bu}\right)_{2}$-Liganden. Die tert-Butylgruppen an den Stickstoffatomen sind insoweit strukturbestimmend, als sie die Konformation des Moleküls festlegen. Sie sind derart ausgerichtet, daß sie größtmöglichen

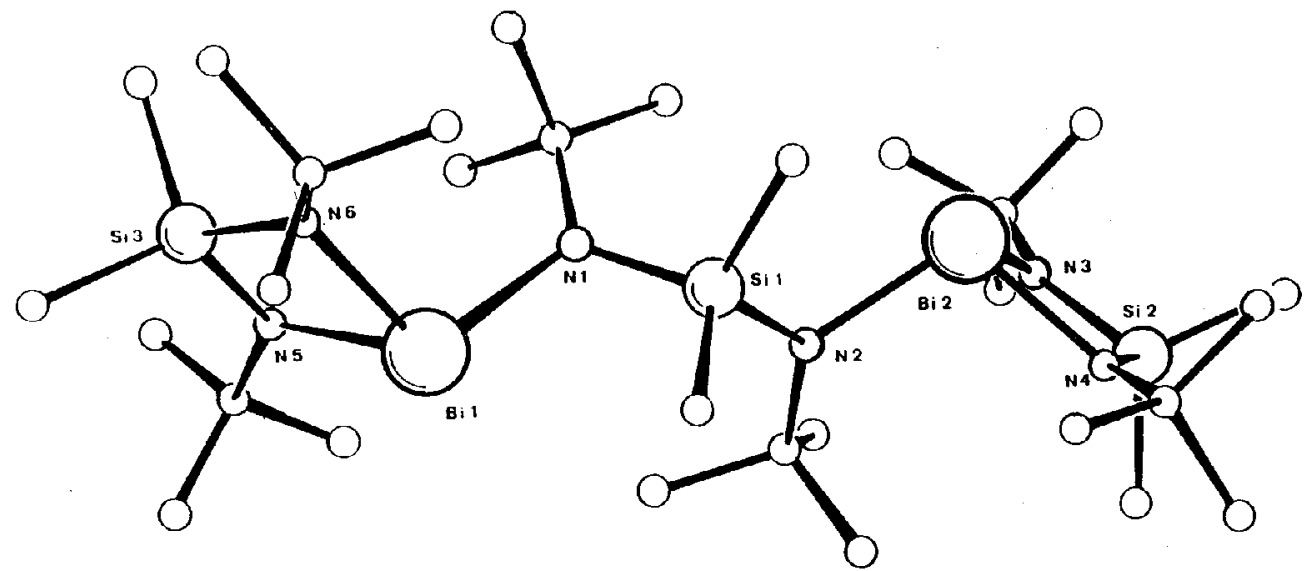

Abb. 3 Zeichnerische Darstellung des Moleküls $\left[\mathrm{Me}_{2} \mathrm{Si}\left(\mathrm{N}^{t} \mathrm{Bu}\right)_{2} \mathrm{Bi}\right]_{2}\left(\mathbb{N}^{t} \mathrm{Bu}\right)_{2} \mathrm{SiMe}_{2}(6)$ mit einigen Atombezeichnungen. 
Tabelle 11. Ausgewählte Bindungslängen (pm) und Winkel (9) im Molekïl

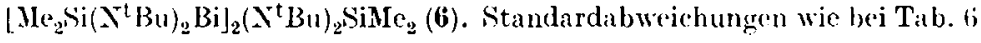

\begin{tabular}{|c|c|c|c|}
\hline$B i(1)-N(5)$ & $216,3(8)$ & $\operatorname{Bi}(\ddot{2})--\mathrm{N}(3)$ & $217,9(8)$ \\
\hline $\operatorname{Bi}(1) \quad X(6)$ & $214,8(9)$ & $B i(2)-N(4)$ & $216,9(9)$ \\
\hline $\mathrm{Bi}(1)-N(1)$ & $2 \cdot 20,2(8)$ & $B i(\dot{y}) \cdot N(\dot{y})$ & $20(1,7(8)$ \\
\hline$N(5) \quad(4)$ & $150(\cdot)$ & $N(3)-C(9)$ & $144\left(\cdot \frac{\cdot}{2}\right)$ \\
\hline$N(6) \cdot C(3)$ & $148\left(\frac{3}{2}\right)$ & $N(\cdot) \quad(10)$ & $143(2)$ \\
\hline$N(5)-S i(3)$ & $170(1)$ & $N(B)-S i(\cdot)$ & $17(0,8(9)$ \\
\hline$N(6) \ldots . S i(3)$ & $171,5(9)$ & $N(4) \quad S i(-))$ & $17 \div(1)$ \\
\hline$\Delta \mathbf{i}(3)--(\cdot(1)$ & $187(\because)$ & $\operatorname{si}(2) \cdot(11)$ & $188(1)$ \\
\hline \multirow[t]{2}{*}{$\operatorname{Si}(3)-(y)$} & $187(*)$ & $\mathrm{Si}(\cdot 2)-\mathrm{C}(1 \cdot 2)$ & $189(\stackrel{\circ}{-})$ \\
\hline & & 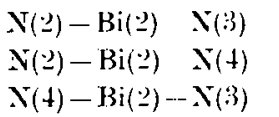 & $\begin{array}{r}109,9(3) \\
105,5(3) \\
70,9(3)\end{array}$ \\
\hline$N(5)-B i(1)-N(1)$ & $110,5(3)$ & & \\
\hline$N(6)-B i(1) \cdot N(1)$ & $104,8(3)$ & 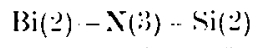 & $96,8(4)$ \\
\hline$N(5) \cdot B i(1) \cdot N(6)$ & $70,6(3)$ & $\begin{array}{l}\mathrm{Bi}(2)-N(3) \quad((9) \\
\mathrm{Si}(2) \quad N(3)-C(9)\end{array}$ & $\begin{array}{l}124,6(7) \\
133,8(8)\end{array}$ \\
\hline $\operatorname{Si}(3) \quad X(5)-B i(1)$ & $97,5(4)$ & $B i(2)-N(4)-C(10)$ & $1.55,6(8)$ \\
\hline $\mathrm{Bi}(1)-\mathrm{N}(5)-(\mathrm{C})$ & $125,9(7)$ & $\mathrm{Bi}(\stackrel{2}{2}) \quad \mathrm{N}(4)-\mathrm{Si}(\stackrel{2}{2})$ & $96,9(4)$ \\
\hline$S i(3)-N(5)-C^{\prime}(4)$ & $133,0(7)$ & 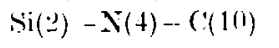 & $13+4.0(8)$ \\
\hline $\operatorname{Bi}(1) \cdots N(6) \quad \mathrm{Ni}(3)$ & $97,7(1)$ & & \\
\hline$S i(3)-N(6)-(1(3)$ & $13 \div, 8(8)$ & & \\
\hline \multirow[t]{2}{*}{$\mathrm{Bi}(1)-\mathrm{N}(6)$} & $1: 25,6(7)$ & & \\
\hline & & $N(3) \cdot S i(2)-N(t)$ & $94, \pi(t)$ \\
\hline$N(5) \quad S i(3)-N(6)$ & $9 ; 3,6(4)$ & $\mathrm{C}(11)-\mathrm{Si}(\stackrel{2}{ }) \quad \mathrm{N}(3)$ & $11+, 5(5)$ \\
\hline$((1)-S i(3)-N(5))$ & $113,8(6)$ & $C(1.1)-\operatorname{Si}(2) \quad N(4)$ & $113,4(6)$ \\
\hline$(C(1)-S i(3)-N(6)$ & $114,4(6)$ & $(C(1 \cdot 2)-S i(\ddot{2}) \quad \mathrm{N}(3)$ & $115.2(6)$ \\
\hline$(\mathrm{C}(2)-\mathrm{Si}(3)-\mathrm{N}(\mathrm{B})$ & $114.2(7)$ & $C(12)-\operatorname{Sit}(2) \quad X(-1)$ & $115,4(6)$ \\
\hline$C\left(\frac{2}{2}\right)-S i(3)-N(6)$ & $113,7(6)$ & $C(11) \quad S i(\cdot 2) \ldots(C(1:)$ & $10+4,1(6)$ \\
\hline$C(1)-\operatorname{Si}(3)-C(2)$ & $106,9(\overline{1})$ & & \\
\hline $\mathrm{Si}(1)-\mathrm{C}(\overline{3})$ & $191(1)$ & & \\
\hline $\mathrm{Si}(1)-C(6)$ & $193(2)$ & & \\
\hline $\mathrm{Si}(1)-\mathrm{N}(1)$ & $173,2(8)$ & & \\
\hline \multirow[t]{2}{*}{$\operatorname{Si}(1)-N(2)$} & $173,5(9)$ & $B i(1)-N(1)-S i(1)$ & $1.13,1(4)$ \\
\hline & & $B i(1)-N(1)-C(\bar{c})$ & $123, \because(6)$ \\
\hline$N(1) \cdots(C(7)$ & $150(1)$ & $C(7)-N(1) \quad S i(1)$ & $123,1(7)$ \\
\hline$N(\stackrel{2}{)}) \cdot(8)$ & $130(1)$ & 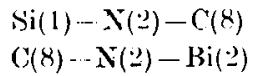 & $\begin{array}{l}1 \cdot 2 \cdot 9(7) \\
1 \cdot 2,1(7)\end{array}$ \\
\hline$N(1)-S i(1)-N(2)$ & $117,3(4)$ & 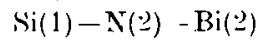 & $1 \cdot 2,7(1)$ \\
\hline$C(5) \quad S i(1)-N(1)$ & $110,5(5)$ & & \\
\hline$C(6)-\operatorname{Si}(1)-N(1)$ & $109,4(5)$ & & \\
\hline$(C(5)-S i(1)-N(2)$ & $1(18,8(\overline{)})$ & & \\
\hline$(C(i)--S i(1)-X(2)$ & $110,2(5)$ & & \\
\hline$(C(5)-\mathrm{Si}(1)-C((6)$ & $99, \geq(6)$ & & \\
\hline $\mathrm{Si}(3) \cdots \mathrm{Bi}(1) \quad \mathrm{N}(1)$ & $114.6(2)$ & & \\
\hline $\operatorname{Si}(3) \cdots \operatorname{Bi}(1)$ & $29 \cdot 2,3(3)$ & & \\
\hline 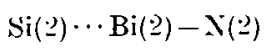 & $11 \bar{j}, 0(2)$ & & \\
\hline $\operatorname{Si}(\ddot{\prime}) \cdots \operatorname{Bi}(\ddot{*})$ & $292,5(3)$ & & \\
\hline
\end{tabular}


Abstand untereinander und zu der Dimethylsilylgruppe des Atoms Si(1) einnehmen. Diese Ausrichtung erfolgt jedoch unter erheblichen Verzerrungen: so beträgt z. B. der Winkel $\mathrm{C}(5)-\mathrm{Si}-\mathrm{C}(6) 99,2^{\circ}$ und die Winkel $\mathrm{N}(1)-\mathrm{Bi}(1) \cdots \mathrm{Si}(3)$ und $\mathrm{N}(2)-\mathrm{Bi}(2) \ldots \mathrm{Si}(2)$ etwa $114,8^{\circ}$, was eine erhebliche Deformation gegenüber dem vergleichbaren Winkel $\mathrm{Cl}-\mathrm{Bi}$...Si in 5 bedeutet (siehe Tab. 9). Einhergehend mit dem Zusammendrücken des $\mathrm{C}(5)$ - $\mathrm{Si}-\mathrm{C}(6)$-Winkels erfolgt eine Aufweitung des $\mathbf{N}(1)-\mathrm{Si}(1)-\mathbf{N}(2)$-Winkels auf $117,3^{\circ}$. Die exocyclischen Bi-N-Abstände sind laut Tab. 11 alle länger als die endocyclischen. Auch dies könnte mit den sterischen Erfordernissen erklärt werden. Die Abstände und Winkel innerhalb der beiden $\mathrm{BiN}_{2} \mathrm{Si}$-Ringe in 6 sind mit den entsprechenden in $5 \mathrm{im}$ allgemeinen vergleichbar. Auffallend ist jedoch, daB die endocyclischen $\mathrm{Bi}-\mathrm{N}$-Abstände in 6 um etwa 4 pm größer sind als in 5 (vgl. Tab. 9 und 11). Andererseits ist die exocyclische $\mathrm{Bi}-\mathrm{N}$-Bindung in 6 prozentual gesehen wesentlich weniger destabilisiert als die $\mathrm{Bi}$-Cl-Bindung in 5. Dieser Befund unterstreicht nochmals, daß die Lockerung der exocyclischen $\mathrm{Bi}-\mathbf{X}$-Bindung im Chlorderivat 5 offenbar mit einer Bindungsverstärkung der $\mathrm{Bi}-\mathrm{N}$-Bindungen einhergeht.

\section{Experimenteller T'eil}

Sämtliche Versuche wurden unter Ausschluß von Luft und Feuchtigkeit unter Stickstoff als Schutzgas in Hochvakuumapparaturen durchgeführt. ${ }^{1}$ H-NMR-Spektren wurden auf einem HitachiPerkin-Elmer R-24-B, $60 \mathrm{MHz}$ bzw. Bruker HFX 80 erhalten. Als Massenspektrometer diente ein Varian MAT CH-7. Die C,H,N-Analysen führte die Firma O. Beller, Göttingen, aus.

\section{1,3-Di-tert-butyl-2,2-dimethyl-4-chloro-1,3,2,4-diazasilarsa- (3), -stiba- (4) und -bismutetidin (5)}

Methode A. Auf $4 \mathrm{~g}(0,0198 \mathrm{~mol})$ Bis(tert-butylamino)dimethylsilan [24] in $10 \mathrm{ml} \mathrm{Hexan}$ werden in einem $100 \mathrm{ml}$ Kolben $24,68 \mathrm{ml}$ einer $15 \%$ igen Lösung von $\mathrm{n}$-Butyllithium in Hexan $(0,0395 \mathrm{~mol})$ innerhalb $1 \mathrm{~h}$ zugetropft. Nach einstündigem Aufkochen unter Rückflu $\beta$ werden jeweils $0,02 \mathrm{~mol}$ der entsprechenden Trichloride in $5 \mathrm{ml}$ Hexan tropfenweise zugeführt, wobei sich die Reaktionslösung erwärmt (im Falle von $\mathrm{BiCl}_{3}$ verwendet man Diethylether und kühlt die Reaktionslösung auf $-40^{\circ} \mathrm{C}$ ). Die Trichloride werden vor der Zugabe über Destillation oder Sublimation gereinigt. Es bildet sich ein Niederschlag, der neben Lithiumchlorid auch Sb-oder Bi-Verbindungen enthält. Nach Abtrennen des Niederschlages werden die Lösungsmittel abkondensiert. Nur 3 und 4 lassen sich zur Reinigung destillieren bzw. sublimieren. 5 gewinnt man durch Umkristallisieren aus Benzol oder Toluol. Ausbeuten: $4 \mathrm{~g}$ an $3(65 \%), 5,6 \mathrm{~g}$ an $\mathbf{4}(80 \%)$ und $5,2 \mathrm{~g}$ an $\mathbf{5}(60 \%)$.

Methode B. $\quad 6,4 \mathrm{~g} \quad(0,02 \mathrm{~mol}) \quad$ 1,3-Di-tert-butyl-2, 2-dimethyl-1,3, 2, 4$\lambda^{2}$-diazasilastannetidin [20] in $25 \mathrm{ml}$ Benzol werden vorsichtig mit einer Lösung von $0,02 \mathrm{~mol}$ des Elementtrichlorides in $25 \mathrm{ml} \mathrm{B \curvearrowright nzol} \mathrm{versetzt.} \mathrm{Die} \mathrm{Reaktionslösung} \mathrm{entfärbt} \mathrm{sich} \mathrm{und} \mathrm{es} \mathrm{fällt} \mathrm{ein} \mathrm{weißer} \mathrm{Niederschlag} \mathrm{aus,}$ der als $\mathrm{SnCl}_{2}$ identifiziert werden kann. Nach ${ }^{1} \mathrm{H}-\mathrm{NMR}$ entstehen bei der Umsetzung mit $\mathrm{AsCl}_{3}$ und $\mathrm{SbCl}_{3} 5$ bzw. $10 \%$ an 1,3-Di-tert-butyl-2, 2-dimethyl-4,4-dichloro-1,3,2,4-diazastannetidin [2]. Nach Abtrennen des Niederschlages und nach Abziehen des Lösungsmittels wird wie unter A aufgearbeitet. Ausbeute: 4,04 $\mathrm{g}$ an $3(65 \%), 6,22 \mathrm{~g}$ an $\mathbf{4}(87 \%)$ und $7,21 \mathrm{~g}$ an $5(81 \%)$.

$\mathbf{C}_{10} \mathbf{H}_{24}$ AsClN ${ }_{2} \mathbf{S i} \quad(310,8 \mathrm{~g} / \mathrm{mol})$ (3). Schmelzpunkt $58^{\circ} \mathrm{C}$, Siedepunkt $65^{\circ} \mathrm{C} / 0,01 \mathrm{Torr}$; Sublimationspunkt $33^{\circ} \mathrm{C} / 0,001$ Torr. Molmasse in Benzol $309 \mathrm{~g} / \mathrm{mol}$. Massenspektrum: Gef. $\mathrm{m} / \mathrm{z}=312,297$, 
ber. für $\mathrm{C}_{10} \mathrm{H}_{24} \mathrm{~N}_{2}{ }^{28} \mathrm{Si}^{75} \mathrm{As} s^{37} \mathrm{Cl}(\mathrm{M})^{+} 312$, für $(\mathrm{M}-15)^{+}+297 .{ }^{1} \mathrm{H}-\mathrm{NMR}$ in Toluol (bei $\left.270 \mathrm{~K}\right): 0,21(1,0, \mathrm{~s}$; $\left.\mathrm{Si}-\mathrm{CH}_{3}\right), 0,45\left(1,0, \mathrm{~s} ; \mathrm{Si}-\mathrm{CH}_{3}\right), 1,12\left(6,0, \mathrm{~s} ; \mathrm{C}-\mathrm{CH}_{3}\right) \mathrm{ppm}$.

ber. $\mathrm{C} 38,65, \mathrm{H} 7,78, \mathrm{~N} 9,01, \mathrm{Cl} 11,41, \mathrm{As} 24,11$

gef. C 39,95, H 7,98, N 9,21, Cl 12,05, As 25,31\%.

$\mathbf{C}_{10} \mathbf{H}_{24} \mathrm{ClN}_{2} \mathrm{SbSi} \quad(357,6 \mathrm{~g} / \mathrm{mol})(4)$. Schmelzpunkt $91^{\circ} \mathrm{C}$, Sublimationspunkt $33^{\circ} \mathrm{C} / 0,0001$ Torr. Molmasse in Benzol $358 \mathrm{~g} / \mathrm{mol}$. Massenspektrum Gef. $\mathrm{m} / \mathrm{z}=358$, 343, ber. für $\mathrm{C}_{10} \mathrm{H}_{24} \mathrm{~N}_{\mathbf{2}}{ }^{28} \mathrm{Si}^{121} \mathrm{Sb}^{37} \mathrm{CI}(\mathrm{M})^{+}+358$, für $(\mathrm{M}-15)^{+}+343 .{ }^{1} \mathrm{H}-\mathrm{NMR}$ in Toluol: $0,24\left(1,0, \mathrm{~s} ; \mathrm{Si}-\mathrm{CH}_{3}\right), 0,46$ $\left(1,0, \mathrm{~s} ; \mathrm{Si}-\mathrm{CH}_{3}\right), 1,13\left(6,0, \mathrm{~s} ; \mathrm{C}-\mathrm{CH}_{3}\right) \mathrm{ppm}$.

ber. C $33,58, \mathrm{H} 6,76, \mathrm{~N} 7,83, \mathrm{Cl} 9,91, \mathrm{Sb} 34,05$

gef. C $33,64, \mathrm{H} 6,92, \mathrm{~N} 7,91$, Cl 9,87 , Sb $34,90 \%$.

$\mathbf{C}_{10} \mathbf{H}_{24}$ BiClN $_{2} \mathrm{Si} \quad(444,8 \mathrm{~g} / \mathrm{mol})(\mathbf{5})$. Schmelzpunkt $164^{\circ} \mathrm{C}$. Molmasse in Benzol $452 \mathrm{~g} / \mathrm{mol}$. Massenspektrum: Gef. $\mathrm{m} / \mathrm{z}=446,431$, ber. für $\mathrm{C}_{10} \mathrm{H}_{24} \mathrm{~N}_{2}{ }^{28} \mathrm{Si}^{209} \mathrm{Bi}^{37} \mathrm{Cl}\left(\mathrm{M}^{+}\right) 446$, für $(\mathrm{M}-15)^{+} 431$. ${ }^{1} \mathrm{H}-\mathrm{NMR}$ in Toluol $(210 \mathrm{~K}): 0,28\left(1,0, \mathrm{~s} ; \mathrm{Si}-\mathrm{CH}_{3}\right), 1,13\left(3,0, \mathrm{~s} ; \mathrm{C}-\mathrm{CH}_{3}\right) \mathrm{ppm}$.

ber. C $27,00, \mathrm{H} 5,44, \mathrm{~N} 6,29$, Cl 7,97, Bi 46,98

gef. $\mathrm{C} 27,16, \mathrm{H} 5,50, \mathrm{~N} 6,17, \mathrm{Cl} 7,57, \mathrm{Bi} 45,60 \%$.

\section{Bis(1,3-di-tert-butyl-2,2-dimethyl-1,3,2,4-diazasilabismutetidino-N-tert-butylamino)- dimethylsilan (6).}

In eine Lösung von 0,01 mol des Dilithiumsalzes von Bis(tert-butylamino)dimethylsilan (siehe vorstehende Vorsehrift A) in $20 \mathrm{ml}$ Hexan/Benzol wird unter Rühren eine Lösung von 2,08 $\mathrm{g}$ $(0,06 \mathrm{~mol})$ Bismuttrichlorid in $20 \mathrm{ml}$ Ether zugetropft. Nach Beendigung des Zutropfens wird vom grauen gebildeten Niedersohlag abfiltriert und die Lösung unter reduziertem Druck eingeengt. Es verbleibt ein oranger Festkörper, der mehrfach mit Hexan extrahiert wird. Aus dem Extraktionsmittel kristallisiert 6 in Form gelber Kristalle beim Einengen. Ausbeute 2,3 g (22\%).

$\mathbf{C}_{30} \mathbf{H}_{72} \mathbf{B i}_{2} \mathbf{N}_{6} \mathbf{S i}_{3} \quad(1019,15 \mathrm{~g} / \mathrm{mol})(\mathbf{6})$. Schmelzpunkt $173^{\circ} \mathrm{C}$. Zersetz. beim Sublimationsversuch. Molmassenbestimmung in Benzol $1052 \mathrm{~g} / \mathrm{mol}$. Massenspektrum: Gef. $\mathrm{m} / \mathrm{z}=409$, ber. für $\mathrm{C}_{10} \mathrm{H}_{24} \mathrm{~N}_{2}{ }^{28} \mathrm{Si}^{209} \mathrm{Bi}^{+}{ }^{409,}{ }^{1} \mathrm{H}-\mathrm{NMR}$ in Benzol 0,21 $\left(1,0, \mathrm{~s} ; \mathrm{Si}-\mathrm{CH}_{3}\right), 0,38\left(1,0, \mathrm{~s} ; \mathrm{Si}-\mathrm{CH}_{3}\right), 0,51(1,0, \mathrm{~s}$; $\left.\mathrm{Si}-\mathrm{CH}_{3}\right), 1,23\left(6,0, \mathrm{~s} ; \mathrm{C}-\mathrm{CH}_{3}\right), 1,81\left(3,0, \mathrm{~s} ; \mathrm{C}--\mathrm{CH}_{3}\right)$ ppm.

ber. C $35,49, \mathrm{H} 7,14, \mathrm{~N} 8,27, \mathrm{Bi} 40,77$

gef. C $35,03, \mathrm{H} 7,12, \mathrm{~N} 8,12, \mathrm{Bi} 40,39 \%$.

\section{Rechnungen}

Die Berechnungen wurden an dem Rechenzentrum der TU Braunschweig bzw. an dem Rechenzentrum der Universität des Saarlandes mit Hilfe des SHELX-Systems [26] durchgeführt.

\section{Literatur}

[1] VEITH, M.: Z. Naturforsch. 33b (1978) 7.

[2] Verth, M. ; Grosser, M. : Z. Naturforsch. 37b (1982) 1375.

[3] VеIтн, M. : Angew. Chem. 99 (1987) 1; Angew. Chem. Int. Ed. Engl. 26 (1987) 1.

[4] VeITH, M.; BerTsCH, B.; HuCH, V.: Veröff. in Vorbereitung.

[5] Wannagat, U.; Bogusoh, E.; Braun, R.: J. Organomet. Chem. 19 (1969) 367.

[6] Scherkr, O. J.; Schmidt, M.: Angew. Chem. 76 (1964) 144; Angew. Chem. Int. Ed. Engl. 3 (1964) 702 .

[7] ABer, E. W.; Bush, R. P.: J. Organomet. Chem. 3 (1965) 245.

[8] Wannagat, U.; Rabet, F.; Inorg. Nucl. Chem. Letters 6 (1970) 155.

[9] Wannagat, U.; Schlingmann, M.: Z. anorg. allg. Chem. 424 (1976) 87. 
[10] Penchuk, A. M.; Khkanovskit, V. A.; Kuplennik, Z. I.; Filonenko, L. P.: J. Gen. Chem. (USSR) 47 (1977) 49.

[11] HASs, D.: Z. Chem. 4 (1964) 185.

[12] Veith, M.; Grosser, M.; Huch, V.: Z. anorg. allg. Chem. 513 (1984) 89.

[13] Cramer, S. E.; ChorutzT, R. J.; Chav, C. H.; Davis, D. W.: Tetrahedron Lett. 9 (1968) 5799.

[14] Levin, C. C.: J. Amer. Chem. Soc. 97 (1975) 5649.

[15] Cherry, W.; Epiotis, N.: J. Amer. Chem. Soc. 98 (1976) 1135.

[16] Bertsch, B.: Dissertation, Universität des Saarlandes, Saarbrücken 1986; in der Arbeit ist die Verbindung 4 in der Raumgruppe Pmnb aufgestellt.

[17] Hamilton, W. C.: Acta Crystallogr. 12 (1959) 609.

[18] Krannich, L. K.; Thewatt, U.; Cook, W. J.; JaIN, S. R.; Sisler, H. H.: Inorg. Chem. 12 (1973) 2304.

[19] WeIss, J.; Eisenhure, W.: Z. Naturforsch. 22b (1967) 454.

[20] Werss, J.; Ersenhuth, W.: Z. anorg. allg. Chem. 350 (1967) 9.

[21] Huheex, J. E.: Inorganic Chemistry: Principles of Structure and Reactivity, $3^{\text {th }}$ ed., Harper \& Row, New York 1983.

[22] Verth, M.; Bärnrghadsen, H.: Acta Crystallogr. B30 (1974) 1806.

[23] WeLLs, A. F.: Structural Inorg. Chem., $5^{\text {th }}$ ed., Clarendon, Oxford 1984, S. $876 \mathrm{ff}$.

[24] Fink, W.: Helv. Chim. Acta 47 (1964) 498.

[25] Veiтh, M.: Angew. Chem. 87 (1975); Angew. Chem. Int. Ed. Engl. 14 (1975) 263.

[26] ShkLdrtck, G.: Program for Crystal Structure Determination, Version SHELX-76.

Bei der Redaktion eingegangen am 11. Juni 1987.

Anschr. d. Verf.: Prof. Dr. M. VeIth, Dr. B. Bertsch, Fachbereich 13; Inst. f. Anorg. Chemie d. Univ. des Saarlandes, D-6600 Saarbrücken 